\title{
VERTICAL UPWARD INTERMEDIATE SCALE TAYLOR FLOW: EXPERIMENTS AND KINEMATIC CLOSURE
}

\author{
Alexander S. Rattner ${ }^{(a)}$, Srinivas Garimella ${ }^{(a)^{*}}$ \\ ${ }^{(a)}$ George W. Woodruff School of Mechanical Engineering \\ Georgia Institute of Technology, Atlanta GA, 30318
}

\begin{abstract}
The vertical upward Taylor flow regime has been extensively studied at the capillary and large channel scale limits. However, flow behavior at the intermediate scale $(5 \lesssim \mathrm{Bo} \lesssim 40$, or $6 \mathrm{~mm} \lesssim D \lesssim 17 \mathrm{~mm}$ for ambient gas-water flows) is comparatively poorly characterized. This regime is fundamentally different because classes of forces conventionally associated with either small or large Bond number flows are all relevant. In this investigation, air-water Taylor-flow experiments are conducted in 6.0, 8.0, and $9.5 \mathrm{~mm}$ diameter tubes. High-speed video data are collected, and automated image analysis algorithms are developed to measure flow parameters including: bubble rise velocity, liquid film thickness, void fraction, and Taylor bubble and liquid slug lengths. New correlations and flow models are developed to predict these parameters at the intermediate scale. Results from this study enable kinematic closure of intermediate scale Taylor flows.
\end{abstract}

\section{KEYWORDS}

Taylor bubble; Slug flow; Intermediate diameter; Transitional flows; High-speed shadowgraphy imaging

\section{INTRODUCTION}

\subsection{Background}

At intermediate liquid and gas flow rates in channels, elongated relatively large diameter bub-

*Corresponding author: Email: srinivas.garimella@me.gatech.edu Phone: 1-404-894-7479

Address: 801 Ferst Drive, Love Building Room 340, Atlanta, GA 30332, USA 
bles translate, separated by full-diameter liquid slugs (Taitel and Barnea, 1990). At high velocities, turbulent shear at the elongated bubble tails can entrain small bubbles in, or aerate, the liquid slugs (Fernandes et al., 1983). Turbulent dispersion may prevent agglomeration of these small slug bubbles (Barnea and Brauner, 1985). At lower velocities, bubbles are not present in the liquid slugs, and the flow pattern is sometimes referred to specifically as elongated bubble or plug flow. In small diameter (capillary) channels, the elongated bubbles tend to be symmetric with rounded noses and tails. Sometimes this configuration is specifically referred to as bubble-train flow (Thulasidas et al., 1995). In the vertical upward (co-flow) configuration, the flow pattern is referred to as Taylor flow, after the bullet shaped bubbles studied by Davies and Taylor (1950).

In vertical upward Taylor flow, elongated rising bubbles fill a major portion of the channel cross-section, and are surrounded by downward flowing (gravitationally driven) annular liquid films on the walls. The interspersed cylindrical liquid slugs rise at the bulk superficial velocity $(j)$, which is slower than the velocity of the Taylor bubbles $\left(U_{\mathrm{b}}>j\right)$. This flow pattern arises in, and is employed in, many engineering applications, including:

- Airlift and bubble pumps - In these devices, rising Taylor bubbles lift liquid, enabling mechanically simple pumping. Relatively high pumping efficiencies can be obtained in the Taylor-flow pattern because liquid only drains downwards in the thin films around elongated bubbles (Delano, 1998). End uses include well dewatering, nuclear fuel reprocessing (de Cachard and Delhaye, 1996), and single-pressure refrigeration systems (von Platen and Munters, 1928).

- Miniaturized heat and mass exchangers - The Taylor-flow pattern has been targeted for monolithic catalytic reactors as a high-interfacial area density, relatively low pressure drop, operating mode (Thulasidas et al., 1995). Additionally, in microchannels, Taylor flow can oc- 
cur for a large portion of the flow length during phase change processes, such as evaporation and condensation (Garimella, 2004).

- Petrochemical processes - Taylor flow of hydrocarbon gases and liquids often occurs in oilwell bores (Fernandes et al., 1983), and it is often necessary to employ "slug catchers" in petrochemical processes to dampen the effects of intermittent flow.

Considering these examples, Taylor flow can occur over a large span of channel diameters ( $\mu \mathrm{ms}$ to $\mathrm{cms}$ ), flow rates, and fluid properties (water to heavy oils). Forces due to inertia (fluid acceleration, turbulence), gravity (buoyancy), friction (within the fluid medium and at the tube wall), and surface tension may thus significantly affect flow behavior. However, comprehensive investigations have thus far been available only at the asymptotic limits where some of these forces are negligible.

Extensive literature is available for Taylor flows in large scale channels. This regime has been defined using criteria based on the Bond number $\left(\mathrm{Bo}=\left(\rho_{\mathrm{L}}-\rho_{\mathrm{G}}\right) g D^{2} / \sigma\right)$, a measure of relative gravitational-to-surface tension force strengths. Here, $\rho_{\mathrm{L}}$ and $\rho_{\mathrm{G}}$ represent the liquid and gas densities, respectively, $g$ is the gravitational acceleration, $D$ is the channel diameter, and $\sigma$ is the fluid surface tension. Viana et al. (2003) identified the following criterion for large-scale flows: Bo $=\left(\rho_{\mathrm{L}}-\rho_{\mathrm{G}}\right) g D^{2} / \sigma \gtrsim 40$ ( $D \gtrsim 17$ mmfor ambient gas-water flow). For such cases, surface tension forces are negligible, leaving flows governed by inertial, viscous, and gravitational forces. Such configurations are of particular interest for petrochemical extraction and separation, and have generally been studied in either the viscous limit (for heavy products) or the inertial (turbulent) limit for high flow rates in large scale channels. Comprehensive flow models for these configurations have been reported by Fernandes et al. (1983), Sylvester (1987), and Taitel and Barnea (1990). Detailed shadowgraphy and particle image velocimetry studies have also been conducted 
to measure Taylor bubble profiles and near-bubble liquid velocity fields in the large scale regime (Bugg and Saad, 2002; Nogueira et al., 2006a, b).

Many investigations have also been performed for Taylor flows at the capillary and microchannel scale. Several criteria have been proposed for defining this regime (Bo $\lesssim 0.9-19.7$ (Khandekar et al., 2010)). This study adopts the condition of Bo $\lesssim 5$, the maximum Bond number for which a Taylor bubble will rise in a stagnant liquid medium (Bendiksen, 1985). In such cases, inertial and gravitational forces are small or negligible compared to viscous and surface tension forces. Applications of interest include monolithic catalytic reactors (Thulasidas et al., 1995), microchannel heat and mass exchangers (Garimella, 2000), and fuel cells (Anderson et al., 2010; Argyropoulos et al., 1999; Hussaini and Wang, 2009). Flow models and literature reviews for Taylor flows at this scale can be found in Thulasidas et al. (1995), Garimella (2004), Liu et al. (2005), and Angeli and Gavriilidis (2008).

In contrast, vertical upward Taylor flows in the intermediate scale regime $(5 \lesssim$ Bo $\lesssim 40)$ where all four classes of forces are relevant, have not yet been well characterized (Reinemann $e t$ al., 1990). At this scale, the Taylor-flow pattern is particularly attractive for gas-lift-pump and bubble-pump applications (de Cachard and Delhaye, 1996).

Analytical or mechanistic Taylor-flow models have been proposed in the literature in which the flow pattern is modeled as identical repeating unit cells of liquid slugs and elongated Taylor bubbles (Fig. 1). Appropriate closure models can then be applied for parameters including bubble rise velocity $\left(U_{\mathrm{b}}\right)$, slug or bubble length $\left(L_{\mathrm{s}}\right.$ and $\left.L_{\mathrm{b}}\right)$, and frictional resistances (Fernandes et al., 1983; Sylvester, 1987; Taitel and Barnea, 1990; Thulasidas et al., 1995). Many correlations have been reported for such closure models, but few are applicable in the intermediate scale regime where all four aforementioned classes of forces can be significant. Additionally, intermediate 


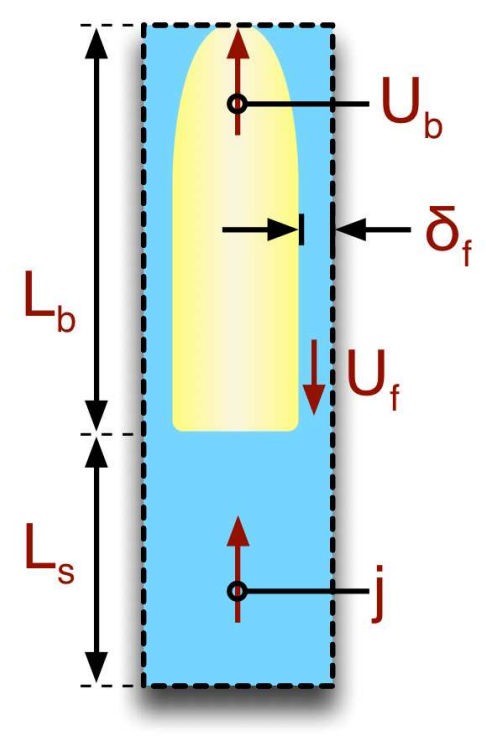

Figure 1 - Repeating unit cell model for Taylor flow

scale Taylor flows tend to span the laminar-to-turbulent transition, indicating strong sensitivity to Reynolds numbers. This investigation focuses on measurement and prediction of the Taylor bubble rise velocity, liquid film thickness, void fraction, Taylor bubble length, and liquid slug length in intermediate scale flows.

\subsection{Prior Work}

\subsubsection{Taylor bubble rise velocity prediction}

In large scale flows, the rise velocity of Taylor bubbles has generally been modeled in a drift flux fashion:

$$
U_{\mathrm{b}}=C_{0} j+\Gamma \sqrt{g D}
$$

The $\Gamma$ term corresponds to the rise velocity of a bubble in a quiescent liquid medium (no net liquid flow). $C_{0}$ is referred to as the distribution parameter, and accounts for the fact that Taylor bubbles rise faster than the bulk flow because the surrounding liquid film drains downward. While the governing fluid mechanics equations are non-linear, this superposition approach for bubble velocity has proven successful in the literature. In general, these models assume that indi- 
vidual Taylor bubbles are long, sufficiently far apart (no drafting, (Barnea and Taitel, 1993)), and that liquid transport properties are dominant $\left(\rho_{\mathrm{G}} / \rho_{\mathrm{L}} \ll 1, \mu_{\mathrm{G}} / \mu_{\mathrm{L}} \ll 1\right.$, where $\mu$ is the dynamic viscosity).

The $\Gamma$ parameter is assumed independent of total flow rate $(j)$; therefore, dimensional analysis indicates that it can be correlated in terms of the Bond number (Bo) and viscous number $\left(\mathrm{N}_{\mathrm{f}}\right)$ (White and Beardmore, 1962).

$$
\text { Bo }=\frac{\left(\rho_{\mathrm{L}}-\rho_{\mathrm{G}}\right) g D^{2}}{\sigma} \quad \mathrm{N}_{\mathrm{f}}=\sqrt{\frac{\rho_{\mathrm{L}}\left(\rho_{\mathrm{L}}-\rho_{\mathrm{G}}\right) g D^{3}}{\mu_{\mathrm{L}}^{2}}}
$$

In the limit of negligible surface tension and viscous forces, $\Gamma$ approaches approximately 0.35 (Davies and Taylor, 1950; Dumitrescu, 1943).

White and Beardmore (1962) found that surface tension effects on $\Gamma$ are negligible for Bo > 70. They proposed the following correlation:

$$
\begin{gathered}
\Gamma=0.345\left[1-\exp \left(\frac{-0.01 \mathrm{~N}_{\mathrm{f}}}{0.345}\right)\right]\left[1-\exp \left(\frac{3.37-\mathrm{Bo}}{m}\right)\right] \\
m=\left\{\begin{array}{cc}
10 & \mathrm{~N}_{\mathrm{f}}>250 \\
69 \mathrm{~N}_{\mathrm{f}}^{-0.35} & 18<\mathrm{N}_{\mathrm{f}}<250 \\
25 & \mathrm{~N}_{\mathrm{f}}<18
\end{array}\right.
\end{gathered}
$$

Bendiksen (1985) modeled the Taylor bubble rise in the limit of negligible viscous forces and relatively small surface tension forces, and proposed the curve fit:

$$
\Gamma=0.486 \sqrt{1+\frac{20}{\mathrm{Bo}}\left(1-\frac{6.8}{\mathrm{Bo}}\right)} \frac{1-0.96 \exp (-0.0165 \mathrm{Bo})}{1-0.52 \exp (-0.0165 \mathrm{Bo})}
$$

This fit was reported to be valid for Bo > 5.7. Bendiksen (1985) also reported that $\Gamma=0$ for Bo 
$<4.5$; i.e., for low Bo (capillary) flow, individual elongated bubbles cannot rise without also effecting a net flow $(j>0)$. Bretherton (1961) found a slightly lower critical Bond number of 3.37. Studies on capillary flows generally assume $\Gamma=0$.

Viana et al. (2003) conducted a survey of experimental studies of Taylor bubble rise velocities in quiescent liquid-filled tubes, and developed a universal empirical correlation for $\Gamma$. The authors found that $\Gamma$ is independent of Bo for Bo $>40$ and that $\Gamma$ rapidly approaches 0 for Bo $<10$. Funada et al. (2005) applied the results of Viana et al. (2003) to predict the rise velocity of elongated ellipsoidal bubbles, and thus estimate Taylor bubble nose profiles.

Mandal et al. (2007) investigated the rise velocity of liquid Taylor bubbles in quiescent higherdensity liquid-filled tubes. The authors developed a correlation for $\Gamma$ accounting for the effects of reduced buoyancy forces.

Results from dimensional analysis indicate that the distribution parameter is a function of the Reynolds number $\left(\mathrm{Re}_{j}\right)$, Bond number (Bo), and capillary number $(\mathrm{Ca})$.

$$
\operatorname{Re}_{j}=\frac{\rho_{L} j D}{\mu_{L}} \quad \mathrm{Ca}=\frac{\mu_{L} j}{\sigma}
$$

In the limit of high $\mathrm{Re}_{j}$ and $\mathrm{Bo} \rightarrow 0$ (i.e. large scale channels), $C_{0}=1.20$ (Nicklin et al., 1962). For low Re with Bo $\rightarrow 0$, Taylor (1961) found that $C_{0}=2.29$. For turbulent flow and Bo $\gtrsim 40$, Bendiksen (1985) proposed the fit:

$$
C_{0}=\frac{\log _{10}\left(\operatorname{Re}_{j}\right)+0.309}{\log _{10}\left(\operatorname{Re}_{j}\right)-0.743}\left\{1-\frac{2}{\mathrm{Bo}}\left[3-\log _{10}\left(\operatorname{Re}_{j}\right) \exp (-0.025 \mathrm{Bo})\right]\right\}
$$

However, this fit does not approach appropriate limits outside the window of applicability, falling below 1.20 for large $\mathrm{Re}_{j}$, and ranging from $-\infty$ to $\infty$ for small Bo. Bendiksen recommended a 
separate laminar fit for $\mathrm{Bo} \lesssim 40$, but the transition between the laminar and turbulent regimes remains an open problem.

$$
C_{0}=2.29\left\{1-\frac{20}{\mathrm{Bo}}[1-\exp (-0.0125 \mathrm{Bo})]\right\}
$$

In the capillary limit $(\mathrm{Bo} \rightarrow 0), C_{0}$ is generally assumed to only be a function of $\mathrm{Ca}$. Fairbrother and Stubbs (1935) developed the implicit correlation:

$$
\frac{\left(1-1 / C_{0}\right)^{2}}{C_{0}}=\mathrm{Ca} \quad 7.75 \cdot 10^{-5}<\mathrm{Ca}<0.014
$$

Liu et al. (2005) developed the following correlation with an extended range of applicability.

$$
C_{0}=\frac{1}{1-0.61 \mathrm{Ca}^{-0.33}} \quad 0.0002<\mathrm{Ca}<0.39
$$

Additional capillary-scale rise velocity correlations can be found in the review of Angeli \& Gavriilidis (2008). In summary, the Taylor bubble rise velocity is well characterized at the asymptotic limits of $\mathrm{N}_{\mathrm{f}}, \mathrm{Bo}$, and $\mathrm{Re}_{j}$, but predictive models validated for intermediate Bond numbers and transitional Reynolds numbers are limited.

\subsubsection{Liquid-film thickness prediction}

In large scale flow models, the thickness of the falling liquid film around Taylor bubbles $\left(\delta_{\mathrm{f}}\right)$ is usually predicted using correlations and analytical results for flat-plate falling-films with no-shear stress interface conditions. This approach is valid in the fully developed film region around long Taylor bubbles. For short bubbles, it may be important to account for the thicker film near the bubble-nose by applying profile models such as those by Dumitrescu (1943), Bendiksen (1985), and Funada et al. (2005).

Fernandes et al. (1983) recommended the Brotz (1952) correlation for the turbulent falling-film 
thickness around long Taylor bubbles. Here, $U_{\mathrm{f}}$ is the average liquid film velocity.

$$
\delta_{\mathrm{f}}\left(\frac{g \rho_{\mathrm{L}}^{2}}{\mu_{\mathrm{L}}^{2}}\right)^{1 / 3}=\left(\frac{3 \mathrm{Re}_{\mathrm{f}}^{2}}{590}\right)^{1 / 3} \quad \operatorname{Re}_{\mathrm{f}}=\frac{\rho_{\mathrm{L}} U_{\mathrm{f}} \delta_{\mathrm{f}}}{\mu_{\mathrm{L}}}
$$

Llewellin et al. (2011) investigated the falling liquid-film thickness around Taylor bubbles in large scale flows (Bo > 40), and developed a correlation for $\delta_{\mathrm{f}}$ as a function of the viscous number $\mathrm{N}_{\mathrm{f}}$. Llewellin et al. (2011) proposed an approach to predict film thickness for intermediate scale Taylor flows, but no experimental data was available for validation.

De Cachard and Delhaye (1996) developed models for Taylor flow in slightly smaller diameter tubes $(10-40 \mathrm{~mm})$. In these cases, the falling films may be laminar or turbulent; thus, a piecewise implicit correlation was recommended:

$$
U_{\mathrm{f}}= \begin{cases}0.333 \frac{g \rho_{\mathrm{L}} \delta_{\mathrm{f}}^{2}}{\mu_{\mathrm{L}}} & \mathrm{Re}_{\mathrm{f}}<750 \\ 15.8\left(g \delta_{\mathrm{f}}\right)^{1 / 2} & \mathrm{Re}_{\mathrm{f}}>750\end{cases}
$$

At the capillary scale, the liquid film still flows downward due to gravity, but its thickness is usually modeled in terms of capillary effects only (independent of channel orientation). Fairbrother and Stubbs (1935) proposed a correlation for liquid film thickness in capillary tubes, which is independent of gravitational forces.

$$
\frac{\delta_{\mathrm{f}}}{D}=0.25 \mathrm{Ca}_{\mathrm{b}}^{1 / 2} \quad \mathrm{Ca}_{\mathrm{b}}=\frac{\mu_{\mathrm{L}} U_{\mathrm{b}}}{\sigma}
$$

Angeli and Gavriilidis (2008) detail a number of other correlations for liquid films in capillary tubes, which are all functions only of Ca. However, Thulasidas et al. (1995) demonstrated that the film thickness in capillary flows is sensitive to orientation. Different trends in $\delta_{\mathrm{f}}$ with orientation were reported at different ranges of $\mathrm{Ca}$. 
It is unlikely that these capillary-number-only correlations apply for intermediate scale flows where inertial forces are significant. The approach of de Cachard and Delhaye (1996) is promising, but has not been validated independently of their overall flow model.

\subsubsection{Void fraction prediction}

Void fraction $(\alpha)$ is a critical parameter for predicting Taylor-flow behavior, particularly in intermediate-to-large scale flows where hydrostatic forces are large relative to frictional forces. In most Taylor-flow models, void fraction is inferred from conservation equations and specified parameters including: liquid and gas flow rates $\left(j_{\mathrm{L}}, j_{\mathrm{G}}\right)$, bubble rise velocity $\left(U_{\mathrm{b}}\right)$, and liquid film thickness $\left(\delta_{\mathrm{f}}\right)$ (Fernandes et al., 1983; Sylvester, 1987; Thulasidas et al., 1995). Prediction of void fraction following this approach requires constitutive models valid for intermediate scale flows.

For large scale flows, dispersed gas bubbles in the liquid slugs can account for a significant portion of the void fraction. Barnea and Brauner (1985) developed a model to predict the gas fraction in liquid slugs, but it is specific to turbulent flows. It is not known whether dispersed bubbles can persist in liquid slugs in intermediate scale flows, where Reynolds numbers typically range from laminar to transitional values.

Many general purpose (flow-regime independent) two-phase void fraction correlations have been reported in the literature. Woldesemayat and Ghajar (2007) performed a rigorous validation study of 68 of these models, but only $4 \%$ of the considered experimental data were from intermediate scale flows.

Microchannel-specific investigations of void fraction were performed by Triplett et al. (1999), Kawahara et al. (2002), and Winkler et al. (2012) (for horizontal minichannels). It is unclear whether any of these correlations apply for vertical upward intermediate scale Taylor flows.

\subsubsection{Taylor bubble and liquid slug length prediction}


The Taylor-flow pattern is often referred to as quasi-periodic (Gao and Jin, 2012) in the sense that there is a repeating series of elongated bubbles and liquid slugs, but individual feature lengths are widely distributed. Even careful injection of identical-size bubbles can lead to downstream bubble trains of widely varying length scales (Nguen et al., 1996). Barnea and Taitel (1993) performed simulations of developing slug flow, and found that the statistical distributions of bubble and slug lengths stabilize sufficiently far downstream. However, general prediction of entrance lengths for slug flow has proven difficult.

In large scale turbulent Taylor flows, liquid slugs are typically many tube diameters $(D)$ in length. Varied representative values are reported in the literature: 4D (Wang et al., 2009), $6-8 D$ (Moissis and Griffith, 1962), 16D (Taitel et al., 1980), 21.7D (Fernandes, 1981), and $30-48 D$ (Schmidt, 1977). Dukler et al. (1985) developed a model to predict the minimum stable liquid slug length as a function of the decaying liquid jet generated at the falling film-to-slug transition. The fact that there is only an apparent physical mechanism for determining the minimum stable slug length may help explain the wide ranges reported in the literature. Taylor bubble lengths are typically not correlated in the large scale tube literature, but rather are inferred from specified liquid-slug lengths and appropriate conservation equations.

Laborie et al. (1999) (Eqn. 14) and Liu et al. (2005) (Eqn. 15) proposed correlations for liquid slug lengths in capillaries in terms of superficial phase velocities and other relevant nondimensional parameters. Laborie et al. (1999) (Eqn. 16) also proposed a correlation for bubble lengths at the same scale.

$$
\frac{L_{\mathrm{s}}}{D}=3451\left(\mathrm{Re}_{\mathrm{G}}^{\prime} \mathrm{Bo}\right)^{-1.2688} \quad \operatorname{Re}_{\mathrm{G}}^{\prime}=\frac{\rho_{\mathrm{L}} U_{\mathrm{b}} D}{\mu_{\mathrm{L}}}
$$




$$
\begin{gathered}
\frac{j_{\mathrm{L}}}{\sqrt{L_{\mathrm{s}}}}=0.088 \operatorname{Re}_{\mathrm{G}}^{0.72} \operatorname{Re}_{\mathrm{L}}^{0.19} \quad \operatorname{Re}_{\mathrm{G}}=\frac{\rho_{\mathrm{G}} j_{\mathrm{G}} D}{\mu_{\mathrm{G}}} \quad \operatorname{Re}_{\mathrm{L}}=\frac{\rho_{\mathrm{L}} j_{\mathrm{L}} D}{\mu_{\mathrm{L}}} \\
\frac{L_{\mathrm{b}}}{D}=0.0878\left(\frac{\operatorname{Re}_{U_{\mathrm{b}}}^{\prime}}{\mathrm{Bo}^{2}}\right)^{0.63} \quad \operatorname{Re}_{U_{\mathrm{b}}}^{\prime}=\frac{\rho_{\mathrm{L}} U_{\mathrm{b}} D}{\mu_{\mathrm{L}}}
\end{gathered}
$$

In all three correlations, relative feature lengths scale with Reynolds number, leading to extreme predictions outside the capillary regime where fitting data were obtained. Additionally, the model of Liu et al. (2005) is dimensionally inconsistent; therefore, its applicability to general Taylor flows is unclear.

Kreutzer et al. (2003) proposed a capillary-scale liquid slug length correlation in terms of void fraction $(\alpha)$.

$$
\frac{L_{\mathrm{s}}}{D}=\frac{1-\alpha}{-0.00141-1.556(1-\alpha)^{2} \ln (1-\alpha)}
$$

While void fraction is generally not known apriori in Taylor-flow models, it is a convenient

parameter for predicting liquid slug length because: $\lim _{\alpha \rightarrow 1} L_{\mathrm{s}}=0$ (gas-only flow) and $\lim _{\alpha \rightarrow 0} L_{\mathrm{s}}=\infty$ (liquid-only flow). However, the correlation of Kreutzer et al. (2003) does not approach the second limit.

Additional correlations for liquid slug, Taylor bubble, and unit cell lengths are discussed by Angeli and Gavriilidis (2008). In general, information about Taylor bubble and liquid slug lengths is not available for intermediate scale flows.

\subsection{Present Investigation}

In the present investigation, vertical upward air-water Taylor-flow experiments are conducted 
with intermediate diameter tubes $(D=6.0,8.0,9.5 \mathrm{~mm}, \mathrm{Bo}=4.9,8.7,12.2)$ for a range of laminar-to-turbulent flow rates $\left(480 \leq \operatorname{Re}_{j} \leq 4460\right.$ ). High speed videos are captured to measure flow parameters including: bubble rise velocity $\left(U_{\mathrm{b}}\right)$, falling film thickness $\left(\delta_{\mathrm{f}}\right)$, void fraction $(\alpha)$, and bubble and slug lengths $\left(L_{\mathrm{b}}, L_{\mathrm{s}}\right)$. Flow models and correlations are developed to predict these parameters for intermediate scale Taylor flows. Additionally, a universal upward-flow Taylor bubble rise velocity correlation is proposed for microchannel-to-large scale flows.

\section{EXPERIMENTAL FACILITY AND PROCEDURE}

\subsection{Facility Overview}

An air-water flow and visualization facility was developed to investigate vertical upward Taylor flows in 6.0, 8.0, and $9.5 \mathrm{~mm}$ ID rounds tubes. A schematic of the experimental facility and photographic configuration is presented in Fig. 2. Tap water at approximately $22^{\circ} \mathrm{C}$ was supplied from a large open reservoir to an inlet port at each tube base. A large diameter $(360 \mathrm{~mm})$ primary reservoir was employed to maintain nearly constant liquid levels and inlet pressures $( \pm 22 \mathrm{~mm}, \pm$ $220 \mathrm{~Pa}$ ) during the experiments.

Compressed air was metered through two needle valves (coarse and fine control), and injected into each tube through a horizontal port $40 \mathrm{~mm}$ above the liquid inlet. Volumetric air flow rates were measured using a Dwyer ${ }^{\circledR}$ rotameter $\left( \pm 38 \mathrm{ml} \mathrm{min}^{-1}\right)$. 

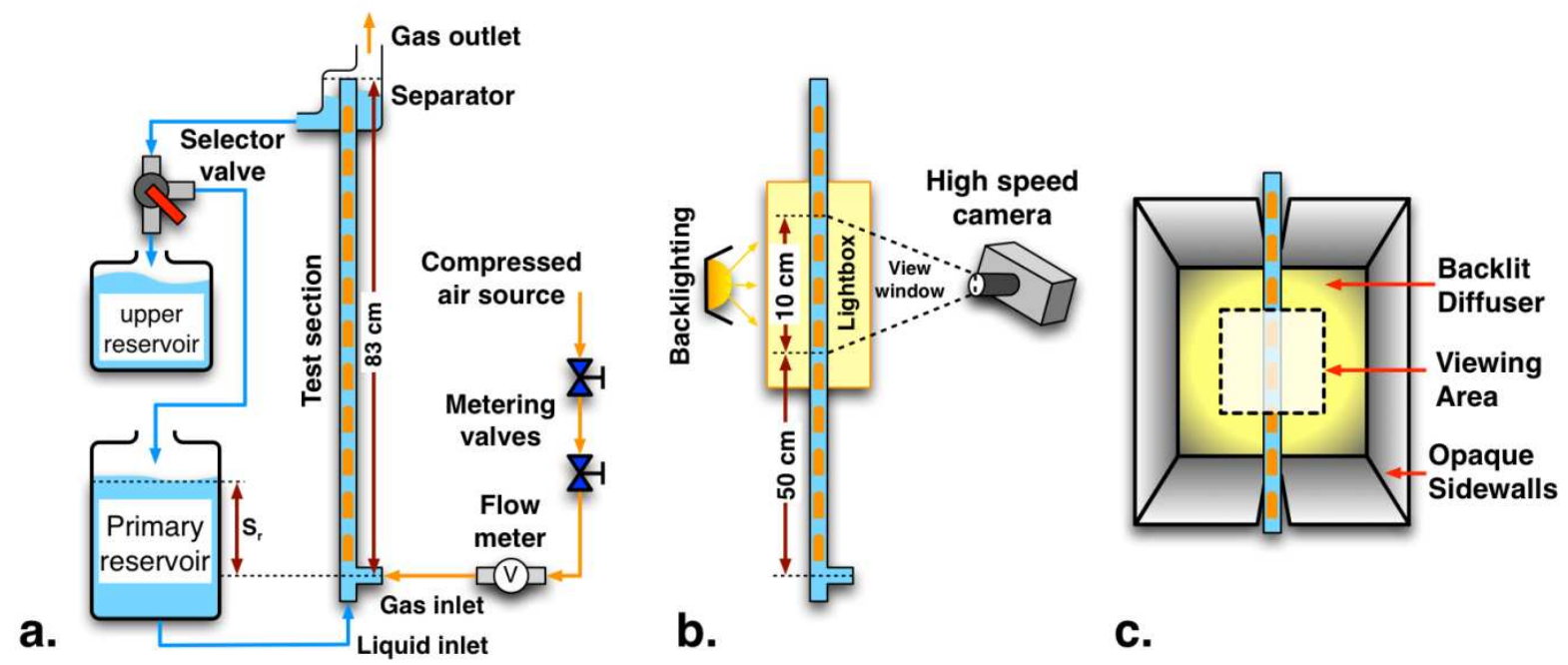

Figure 2 - a. Schematic of experimental facility, b. Photographic and lighting configuration, c. Detail view of lightbox and viewing area

The flow tubes were fabricated from glass to permit visual access, and were approximately 830 $\mathrm{mm}$ in length above the gas inlet. Each flow tube opened to a larger diameter $(22 \mathrm{~mm}) \mathrm{settling}$ section. Water flow from the settling section was diverted to an upper reservoir, which could be weighed to determine average liquid pumping rates during operation. A high speed camera was employed to record flow phenomena during experiments. The camera focus was centered approximately $550 \mathrm{~mm}$ above the gas inlet ports, and the viewing area included approximately $100 \mathrm{~mm}$ of tube length. A small lightbox enclosure was installed around the visualized portions of the test sections. This lightbox was an approximately $0.15 \mathrm{~m}$ cube with an open front for optical access, opaque diffuse side- and top/bottom- walls to prevent glare from outside lighting, and a translucent diffuser-sheet back wall to provide uniform backlighting in the viewing window (Fig. 2c). An optical analysis of this visualization configuration is summarized in Appendix B, and it demonstrates that distortion effects are not significant in this study.

\subsection{Experimental procedure}

Flow experiments were conducted for all tubes at submergence ratios (ratio of reservoir level 
to tube length, $S_{\mathrm{r}}$ ) of $0.3,0.4$, and 0.5 . For each of these conditions, the air flow rate was varied from 142 - $940 \mathrm{ml} \mathrm{min}^{-1}$, although net liquid flow was only achieved above threshold air flow rates in the 8.0 and $9.5 \mathrm{~mm}$ ID tubes at lower $S_{\mathrm{r}}$ values. Measurements were collected for 108 different conditions of tube diameter, $S_{\mathrm{r}}$, and air flow-rate.

For each test condition, the flow was allowed to stabilize, and the settling section liquid outlet was then diverted to the upper reservoir. Air flow was maintained for $4-15$ minutes $( \pm 3$ s estimated uncertainty), and the average liquid pumping rate was determined from the change in upper reservoir mass (measured with a scale with $\pm 1 \mathrm{~g}$ uncertainty). Shorter experimental durations were employed for high liquid flow rate cases in order to maintain the main reservoir level within $22 \mathrm{~mm}\left( \pm 0.027 S_{\mathrm{r}}\right.$, or $\pm 220 \mathrm{~Pa}$ in hydrostatic inlet pressure). The resulting uncertainties in the average liquid flow rates were slightly higher for the shorter duration experiments, but were maintained below $\pm 4 \mathrm{ml} \mathrm{min}^{-1}$. The relative uncertainties were less than $1 \%$, except for unsteady very low flow rate cases $\left(<15 \mathrm{ml} \mathrm{min}^{-1}\right.$ liquid flow). Measured volumetric liquid pumping rates $\left(V_{\mathrm{L}}\right)$ for all tested tube diameters, submergence ratios, and volumetric air flow rates $\left(V_{\mathrm{G}}\right)$ are presented in Fig. 3.

Three 5 s duration high-speed videos (125 FPS) were collected during each experiment to enable evaluation of quantities including bubble rise velocity, liquid film thickness, void fraction, Taylor bubble length, and liquid-slug length. 

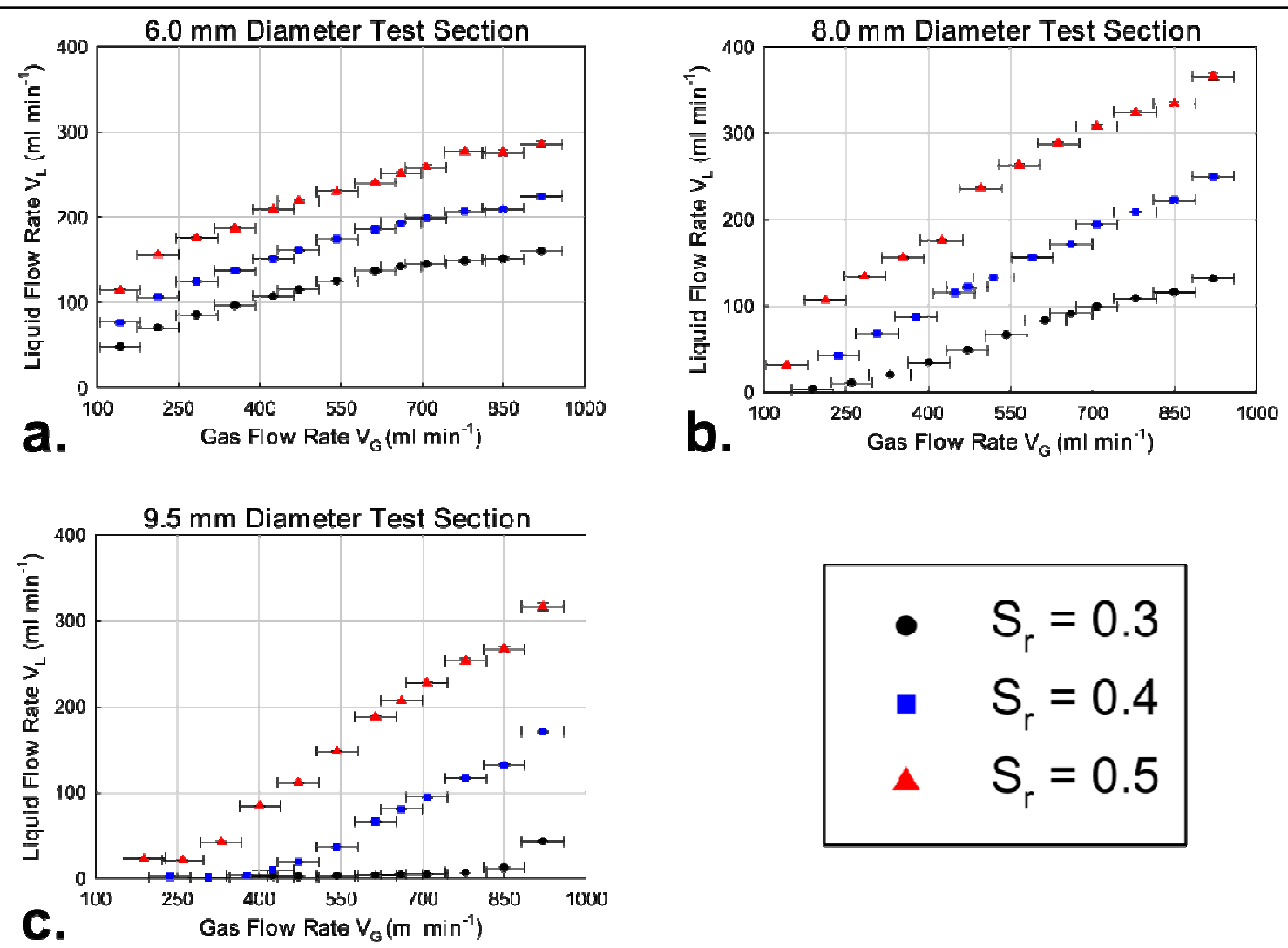

$$
\begin{array}{ll}
\text { - } & S_{r}=0.3 \\
\text { - } & S_{r}=0.4 \\
\text { - } & S_{r}=0.5
\end{array}
$$

Figure 3 - Measured flow rates at submergence ratios of $0.3,0.4$, and 0.5 for: a. $6.0 \mathrm{~mm}$, b. $8.0 \mathrm{~mm}$, c. $9.5 \mathrm{~mm}$ diameter test sections

\section{HIGH-SPEED VIDEO ANALYSIS}

\subsection{Bubble rise velocity measurement}

For each set of recorded flow videos, brief (1 s) no-flow reference videos were collected with the test section entirely filled with air (gas-only) and with water (liquid-only). The glass-tube inside wall edges appeared sharp in the gas-only videos, enabling automated image cropping and rotation correction. The liquid-only reference images are compared with flow images to assist in identifying all-liquid pixel rows and thus, liquid slugs. The following automated procedure was employed to determine the average Taylor bubble rise velocity for each flow experiment.

First, the all-gas reference image series was averaged to correct for camera vibration and lighting variation, and cropped horizontally to the middle 50\%. The top and bottom 100 pixel rows 
were summed vertically, yielding two horizontal signals with sharp minima at the inner tube edges. Midpoints were determined between these upper and lower minima, and the resulting offset was used to rotate the tube images to be vertical. Typical corrective rotation angles were less than 1 degree, but even slight image misalignment could cause tube edges to be included in the search window for liquid-gas interfaces. Tube inside wall positions were then identified as the minimum average intensity image columns in the rotated gas-only images. The average gas-only and liquid-only reference images were then rotated and cropped to these inner tube extents (Figs. $4 a, 4 b)$.

Ten flow images were then loaded, averaged, rotated, and cropped for each flow condition. Correlations (i.e. inner products) were evaluated between these flow and gas-only images with slight horizontal shifts applied. This step enabled automated correction of slight changes in camera orientation during experiments. The optimal (maximum-correlation value) horizontal corrections were generally less than 2 pixels. Finally the full set of images for each flow case was rotated, shifted, and cropped to the inside tube diameter (Fig. 4c).

For each flow image, the left- and right- bubble-interface locations were determined for each row as the minimum intensity pixels on either side of the tube central axis (Fig. 4d). An optical simulation was conducted to validate this interface identification technique and demonstrate that distortion effects were negligible (Appendix B). Slug bubbles were rarely observed in this experimental study; therefore, image rows were classified to be either containing a Taylor bubble or all liquid.

All-liquid rows were identified by comparison with the liquid-only calibration images. This stage was also performed in an automated fashion by evaluating the following heuristic. 


$$
G^{*}=\left|\frac{\Delta(\text { Flow }- \text { Liq })}{\Delta x}\right|+\left|\frac{\Delta(\text { Flow }- \text { Liq })}{\Delta y}\right|
$$

Here, "Flow" and "Liq" refer to the current flow image and averaged liquid-only calibration image, respectively. The $\Delta() / \Delta x$ and $\Delta() / \Delta y$ operations are the finite differences in the horizontal and vertical directions, respectively. The number of $G^{*}$ values more than one standard deviation from the mean of $G^{*}$ was then counted for each row. Image rows with fewer than five such pixels were empirically classified as all liquid. This process sometimes generated mislabelled rows; therefore, a multi-pass interface closing routine (based on neighbor row liquid/gas states) was then applied (Fig. 4e).

This approach still occasionally mislabeled liquid and bubble rows; therefore, an additional heuristic was applied to identify the vertical end positions of Taylor bubble and liquid slug regions. The middle horizontal $50 \%$ of flow images were summed across rows, and the resulting vertical 1-D signal was smoothed. Entries in this signal sufficiently far from the mean (more than 750 in this study) were found to correspond to bubble-slug transition regions. Single row transition regions were discarded as they almost always resulted from image noise. The center points of each remaining transition region were then reported as bubble/slug region ends (Fig. 4f). Intermediate regions with majority all-liquid rows were then recorded as liquid slugs, and remaining regions as containing Taylor bubbles. Observed bubbles were found to almost always be elongated; thus, short regions $(L / D<0.5)$ were always classified as liquid slugs. Occasionally, Taylor bubbles where observed to rise with head-to-tail nearly touching. These were considered to represent single Taylor bubbles in this study as the intermediate slugs where most probably too short to affect the flow dynamics. 


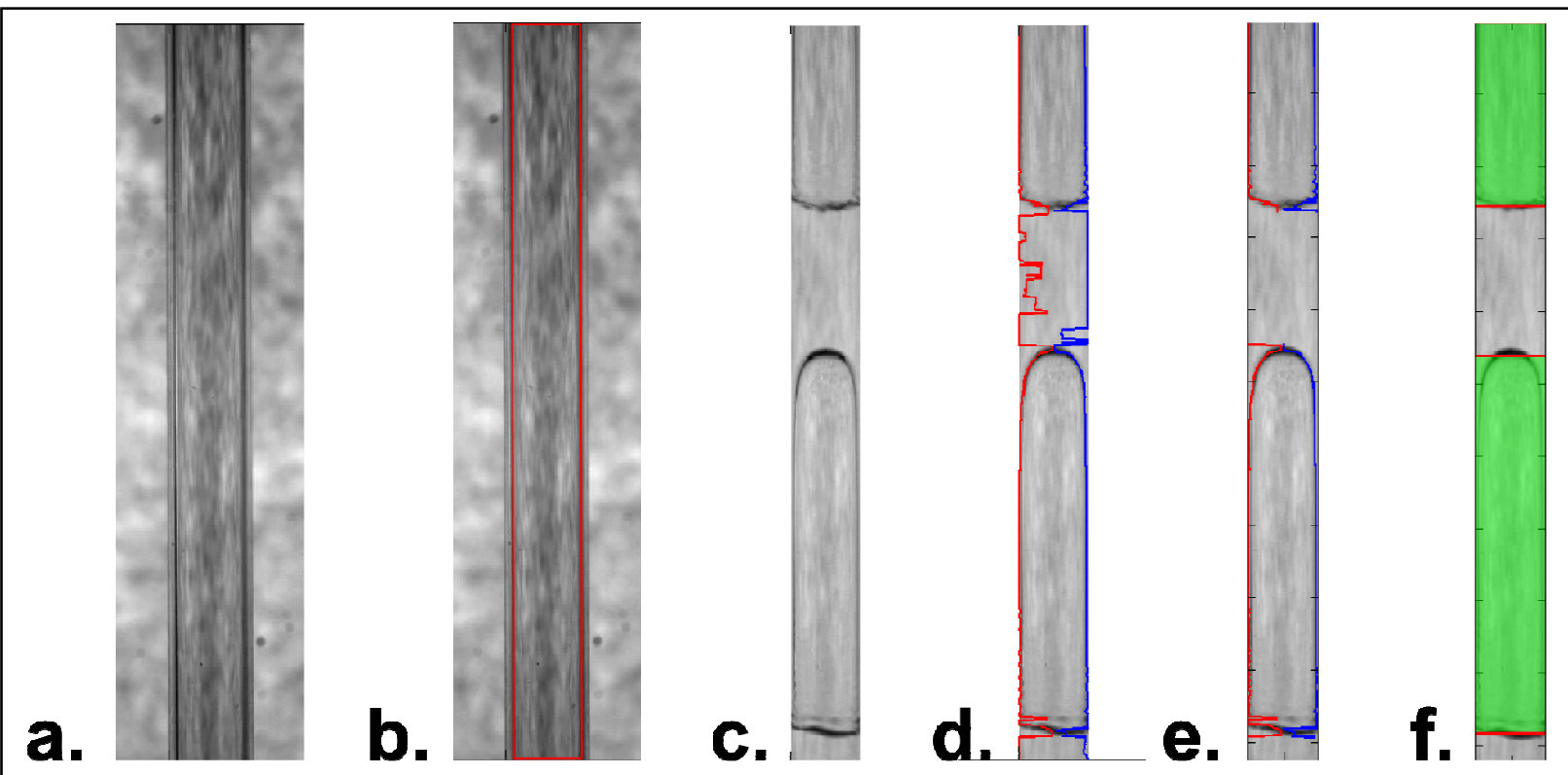

Figure 4 - Automated flow image analysis process: a. Raw calibration image, b. Rotation and cropping window, c. Corrected flow image, d. Interface identification, e. Liquid layer detection and interface closing, f. Bubble and slug segment identification

Bubble rise velocities were then measured using the following automated process. In a first pass, closest bubble nose positions in consecutive video frames were paired. The average instantaneous bubble velocity was then evaluated based on the displacement between these paired positions. This average velocity was then used to extrapolate expected bubble nose positions around which to search in a second pass. This approach was employed to minimize aliasing, wherein trains of consecutive short bubbles could be incorrectly tracked at low video frame rates. Bubbles with noses above the lower $85 \%$ of the camera window were ignored because of the difficulty of estimating the velocity of bubbles that partially exit the view between frames.

Only every $15-100^{\text {th }}$ frame pair was analyzed (18 - 125 frames per test case), depending on the average flow rate. This striding (only evaluating every $n^{\text {th }}$ frame) was performed in order to avoid double counting the velocities of individual bubbles. The reported average velocities are limited such that $U_{\mathrm{b}} \geq j$. This limiting was primarily performed for $6 \mathrm{~mm}$ tube diameter cases where 


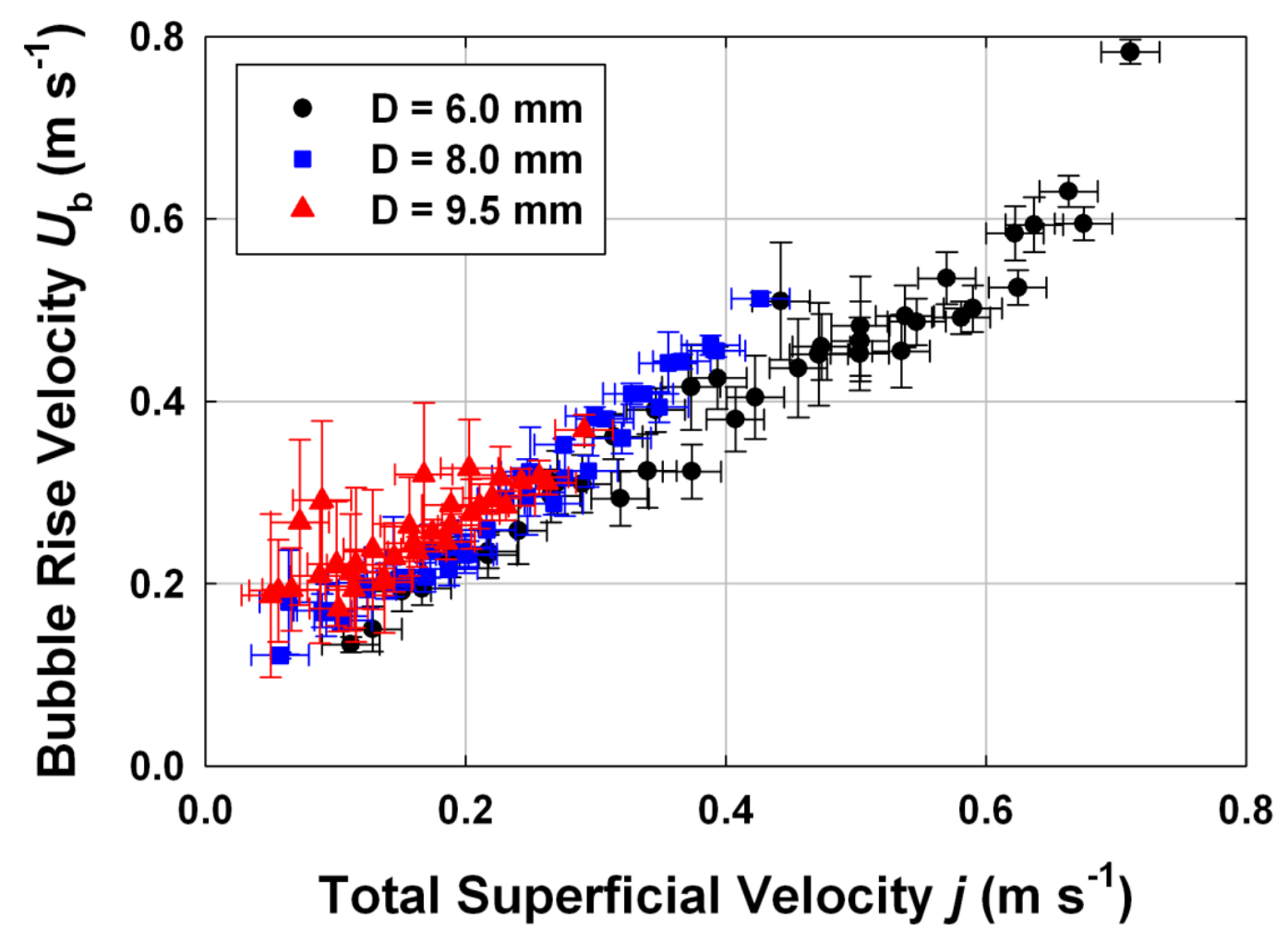

Figure 5 - Average measured Taylor bubble rise velocities

the expected bubble velocities are close to total superficial velocities, and measured values were occasionally less than $j$ (within the measurement uncertainty). Uncertainties are reported as 95\% confidence intervals, and are estimated as 1.96 standard deviations from the mean of the discrete bubble velocity measurements. It should be noted that the reported velocities are numberaveraged (i.e., $\overline{U_{\mathrm{b}}}=\sum_{\mathrm{i}=1}^{\mathrm{n}} U_{b, i} / \mathrm{n}$ ), and might not correspond to the dynamic averages for which uniform-velocity bubbles would yield equivalent flow mechanics (e.g., $\overline{U_{\mathrm{b}}}$ for pressure drop such that $\left.\nabla P_{\text {model }}\left(\overline{U_{\mathrm{b}}}, \ldots\right)=\nabla P_{\text {experiment }}\right)$. Average bubble velocities for all test conditions are presented in Fig. 5.

The observed Taylor-bubble rise-velocity trends are relatively intuitive, with $U_{\mathrm{b}}$ increasing 
nearly linearly with superficial velocity $(j)$. Additionally, larger diameter tubes permit more interphase slip, and thus yield greater bubble rise-velocities for fixed superficial velocities.

An instability was observed in the $6 \mathrm{~mm}$ tube at lower flow rates when successive rising short bubbles periodically merged. The merged-bubble velocity was greater than that of the constitutive bubbles, leading to a runaway process of capture of slower leading bubbles and acceleration until the flow tube was nearly emptied of gas. The resulting high velocity bubbles were longer than the camera window $(\sim 12 D)$; therefore, velocities could not be determined by nose tracking. As a result, reported average rise velocities might be skewed low in these cases, because no values could be obtained for these high-velocity bubbles. This phenomenon was not considered an entranceeffect because it was only observed in the $6 \mathrm{~mm}$ ID flow tube $(L / D=139)$, and not in the relatively shorter $8 \mathrm{~mm}(L / D=103)$ and $9.5 \mathrm{~mm}(L / D=89)$ flow tubes. A comparison of average instantaneous bubble velocities (for each video frame) is presented for one such unstable case and a similar $8 \mathrm{~mm}$ diameter case in Fig 6 (both at $V_{\mathrm{G}}=708 \mathrm{ml} \mathrm{min}^{-1}$ and $S_{\mathrm{r}}=0.4$ ). The mechanics of this flow instability are beyond the scope of the present investigation, and represent an open area for future research in two-phase flow. 


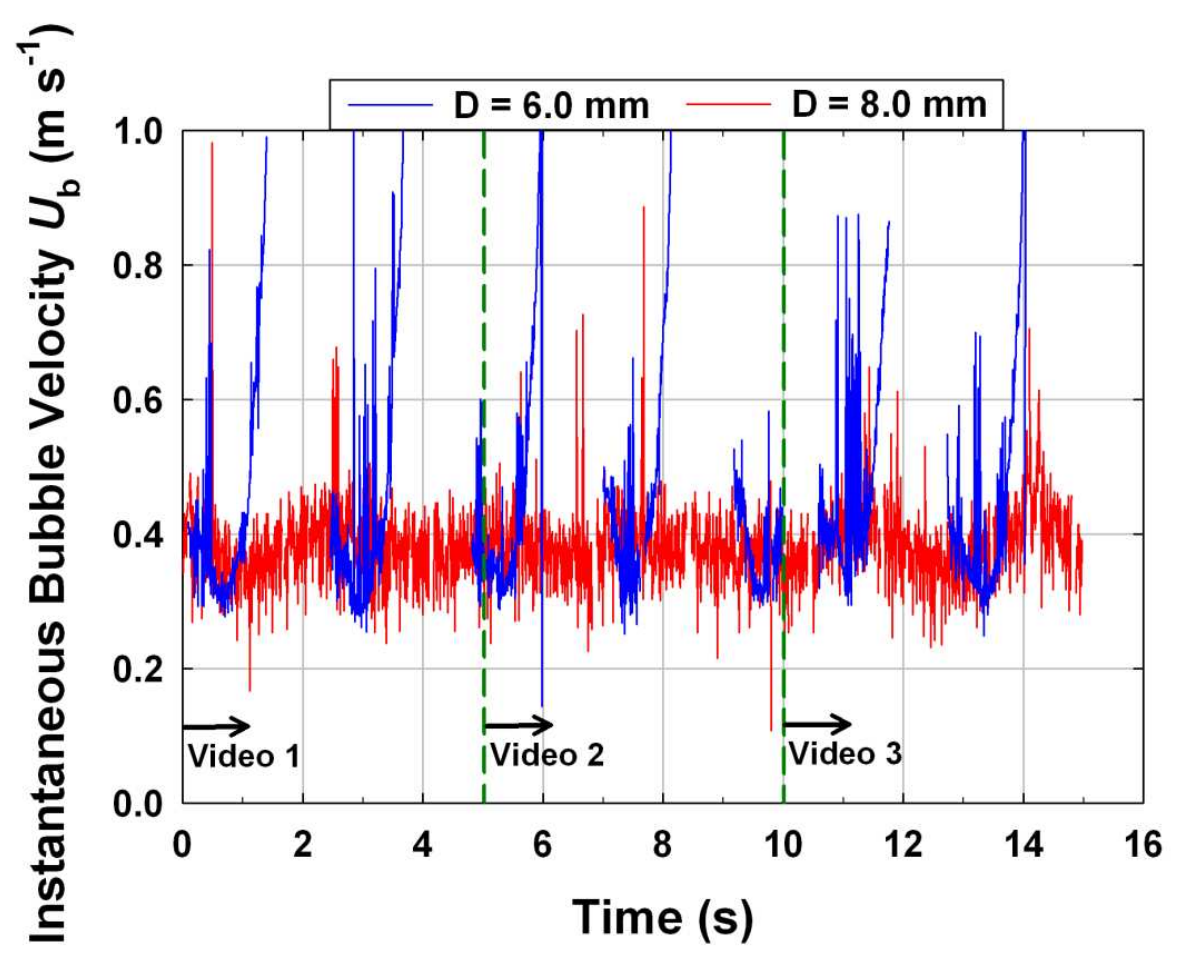

Figure 6 - Instantaneous average bubble velocity measurements for similar conditions in 6.0 and $8.0 \mathrm{~mm}$ diameter tubes $\left(V_{\mathrm{G}}=708 \mathrm{ml} \mathrm{min}^{-1}, S_{\mathrm{r}}=0.4\right)$. The bubble risevelocity instability is apparent for the $6.0 \mathrm{~mm}$ case.

\subsection{Liquid-film thickness measurement}

The process for measuring liquid film thickness began using discrete bubbles and interface positions identified from strided images, following the approach of Section 3.1. The top $1 D$ and bottom $0.5 D$ of each Taylor bubble were cropped to prevent nose and tail sections from skewing average film thicknesses. Reported film thicknesses are total-length-averaged from Taylor bubbles in the strided image series. The experimental facility was not originally optimized for film thickness measurements, so the interface identification uncertainty ( \pm 1 px for $2-5 \mathrm{px}$ thick films) leads to relatively high film thickness uncertainties $(\sim 20-60 \%)$. Measured films thicknesses are presented in Fig. 7. 


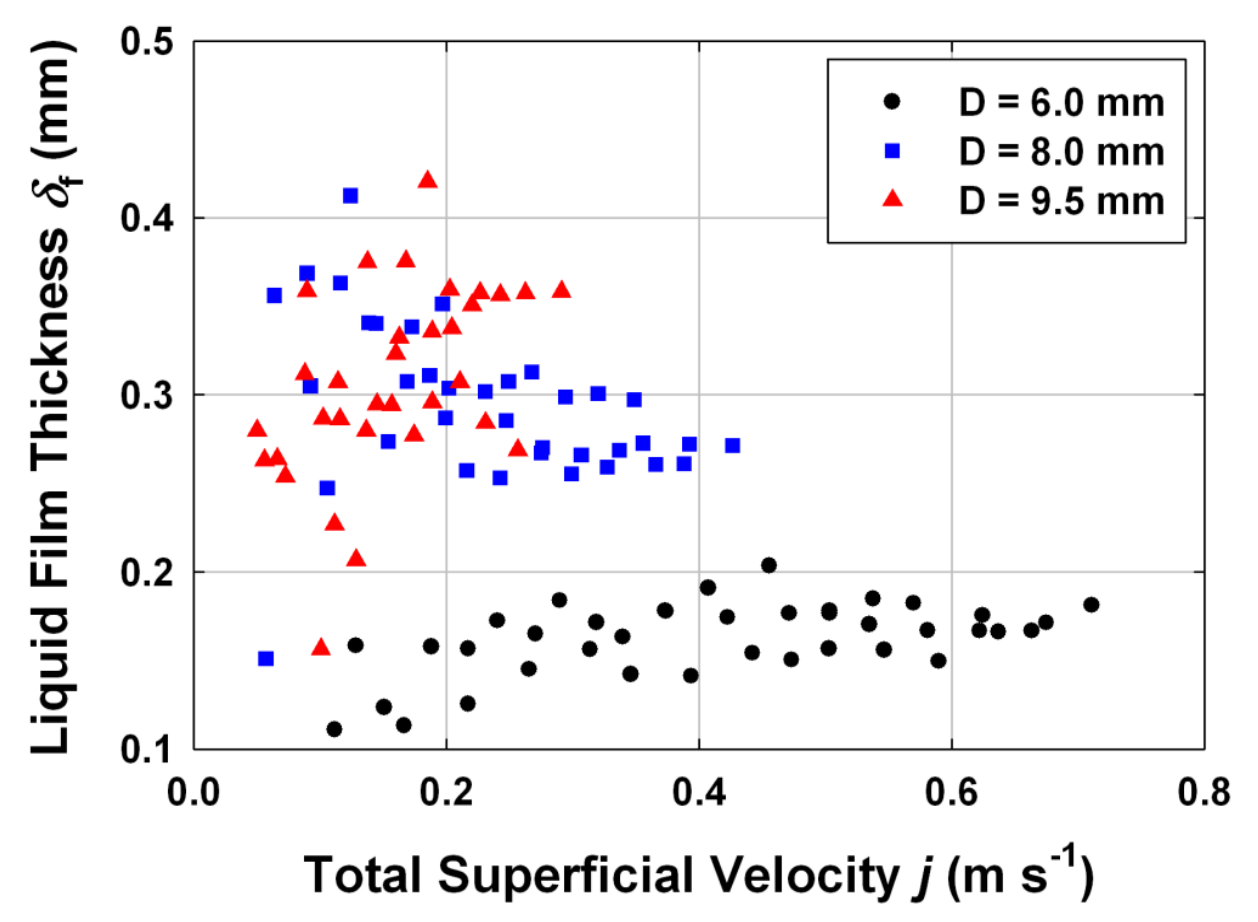

Figure $7-$ Average measured liquid-film thickness $( \pm 0.07 \mathrm{~mm}$ for $D=6.0 \mathrm{~mm}, \pm 0.09$ $\mathrm{mm}$ for $D=8.0 \mathrm{~mm}, \pm 0.08 \mathrm{~mm}$ for $D=9.5 \mathrm{~mm}$ )

Measured film thickness values appear to be nearly independent of flow rates and submergence ratios, and depend primarily on tube diameter. This result supports the gravity-driven falling-film model of de Cachard and Delhaye (1996) rather than the flow-rate (capillary number) dependence typically assumed in capillary-scale flows.

\subsection{Void fraction measurement}

The void fraction measurement process began using discrete bubbles and interface positions obtained following the approach in Section 3.1. Unlike bubble velocity or film thickness, void fraction can meaningfully be time and space averaged. Therefore, data from every video frame and for the full height of each frame was considered. The gas volume on each bubble-pixel row was evaluated assuming cylindrical sections with diameter equal to the left-to-right interface position differences. Averaged void fraction values were obtained for $15 \mathrm{~s}$ of video data (1875 frames) 


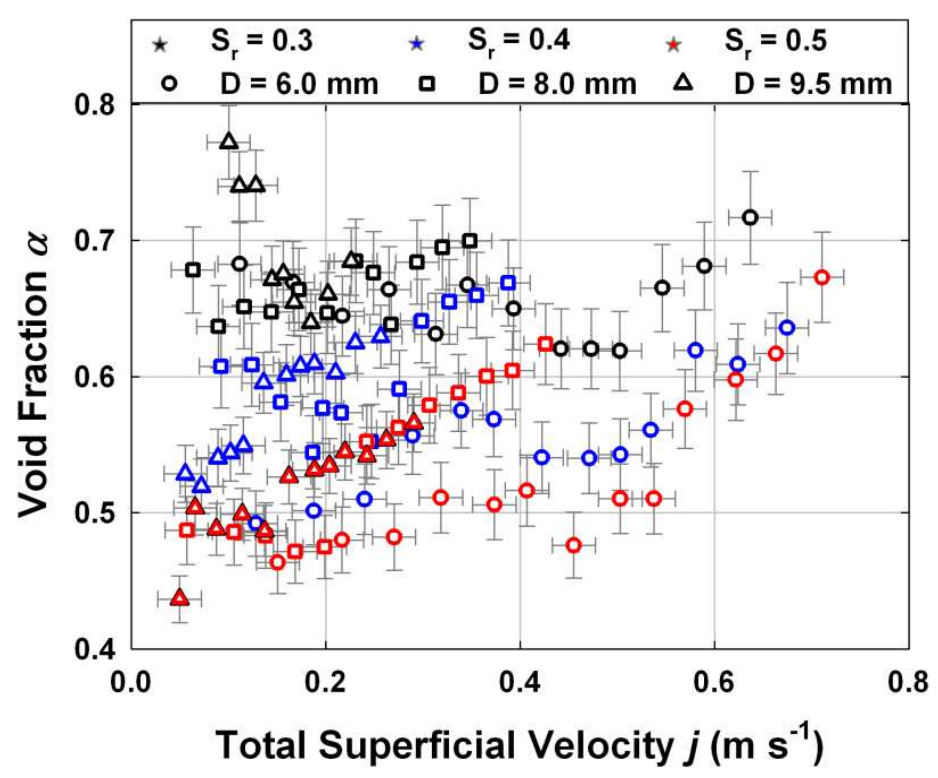

Figure 8 - Time- and space- averaged measured void fractions

for each test condition. In the recorded videos, the liquid-gas interfaces appear to span $\sim 2$ pixels. Upper and lower void-fraction bounds were obtained by offsetting the interface locations on each row by one pixel outward and inward, respectively. The resulting uncertainties are typically 3 $6 \%( \pm 0.02-0.03)$ of the averaged void fraction values, which range from $0.44-0.77$. Evaluated average void fractions for all experimental conditions are presented in Fig. 8.

It is difficult to discern overall trends in void fraction from inspection of these experimental results. This is because the void fraction is a composite result of multiple factors including: Taylorbubble rise velocity, liquid-film thickness, and liquid and gas flow rates. Thus, in Section 4.3, a comprehensive kinematic flow model is employed to predict void fraction as a function of these parameters.

\subsection{Taylor bubble and liquid slug length measurement}

The feature length measurement process begins by collecting discrete bubble and slug data from strided images following the approach in Section 3.1. Only bubbles and slugs at least partially inside the vertical middle $70 \%$ of the camera window were considered, reducing the need to 
extrapolate the length of features extending outside the camera window. Some long features were still observed extending from the middle window to outside the overall camera window. In particular, some very long Taylor bubbles were observed in the $6 \mathrm{~mm}$ ID tube flows. Such features were assumed to be twice as long as their extents within the camera window, or potentially 4 times as long if they extended beyond the top and bottom of the camera window. This approach can be considered unbiased, because long features should be equally likely to be $x \%$ or (100-x)\% within the camera window at a given time.

Measured bubble and slug lengths were observed to follow an approximately log-normal statistical distribution, in agreement with the results of Brill et al. (1981), Barnea and Taitel (1993), and Wang et al. (2009). Representative length histograms for an $8.0 \mathrm{~mm}$ diameter tube flow case are presented in Fig. 9. These histograms incorporate data for 78 Taylor bubbles (Fig. 9a) and 43 liquid slugs (Fig. 9b).

Average bubble and slug length measurements for all cases are presented in Fig. 10. Uncertain-
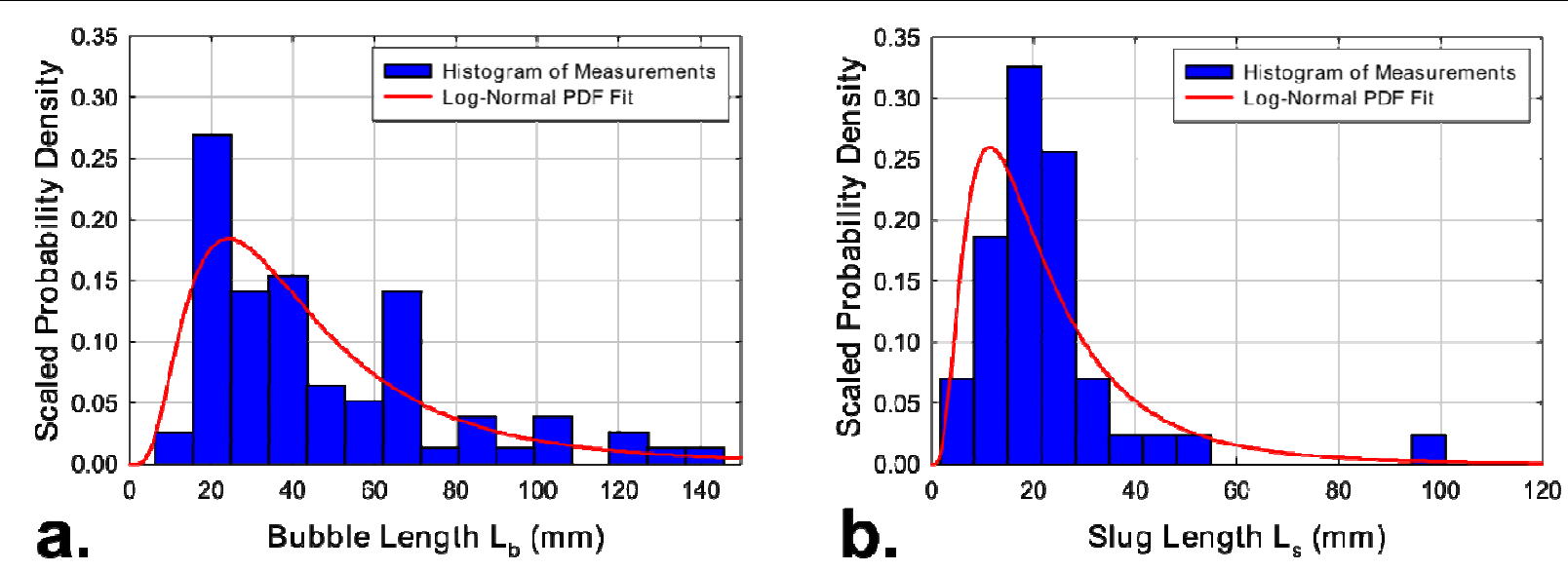

Figure 9 - Representative feature length distributions and best-fit log-normal PDF fits scaled by histogram bin size $\left(D=8.0 \mathrm{~mm}, V_{\mathrm{G}}=708 \mathrm{ml} \mathrm{min}^{-1}, S_{\mathrm{r}}=0.4\right)$. a. Taylor bubble length distributions (9.3 $\mathrm{mm}$ bins), b. Slug length distributions (6.6 mm bins) 

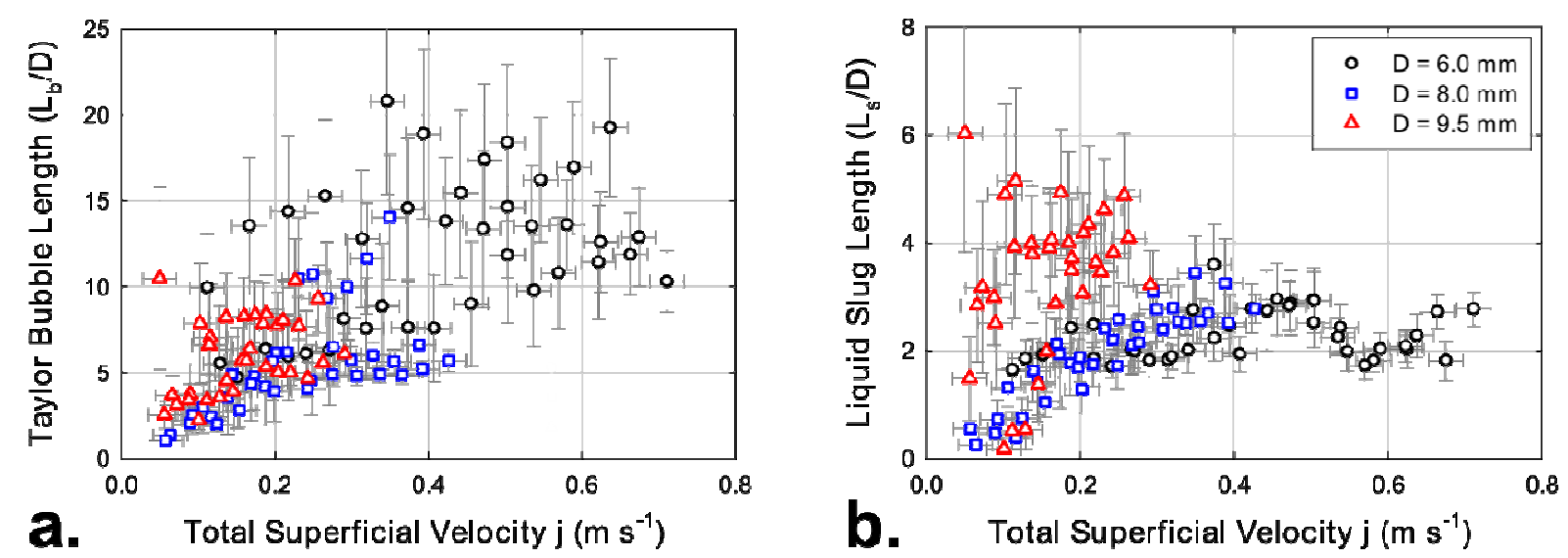

Figure $10-\quad$ Average measured Taylor bubble (a) and liquid slug (b) lengths

ties are reported as $95 \%$ confidence intervals, which are evaluated as 1.96 standard deviations from the mean of the discrete length measurements. Bubble length trends are apparent, but slug lengths of $1-6 D$ are found for nearly all cases.

The complete set of measured parameters $\left(S_{\mathrm{r}}, \nabla P\right.$ - pressure drop gradient, $V_{\mathrm{G}}, V_{\mathrm{L}}, U_{\mathrm{b}}, \delta_{\mathrm{f}}, \alpha$, $\beta$ - relative Taylor bubble to unit-cell length, $L_{\mathrm{b}}$, and $L_{\mathrm{s}}$ ) is summarized for reference in Appendix A.

\section{FLOW PARAMETER MODELING AND CORRELATION}

\subsection{Bubble rise velocity prediction}

The Taylor bubble rise velocity model proposed in this study follows the drift-flux approach employed in previous investigations in which:

$$
U_{\mathrm{b}}=C_{0}\left(\operatorname{Re}_{j}, \mathrm{Bo}, \mathrm{Ca}\right) j+\Gamma\left(\mathrm{Bo}, \mathrm{N}_{\mathrm{f}}\right) \sqrt{g D}
$$

At the low-viscosity limit, Bendiksen (1985) reported a curve fit to an exact solution for $\Gamma$ (Bo) valid for Bo $\gtrsim 5.7$. Here, this curve fit is extended to the limit of Bo $\geq 4.55$, below which it is 0 (no bubble rise without net flow). Additionally, the low-viscosity limit $\left(\mathrm{N}_{\mathrm{f}}>250\right)$ exponential correction factor from White and Beardmore (1962) is applied. However, this term could not be 
independently validated in this study because this viscous effect was found to be negligible for all considered cases except for capillary flow cases where Bo $<4.55$, setting $\Gamma=0$.

$$
\Gamma=\left\{\begin{array}{cc}
0.344\left[1-\exp \left(\frac{-0.01 \mathrm{~N}_{\mathrm{f}}}{0.345}\right)\right] \sqrt{1+\frac{20}{\mathrm{Bo}}-\frac{93.7}{\mathrm{Bo}^{2}}-\frac{676.5}{\mathrm{Bo}^{3}}+\frac{2706}{\mathrm{Bo}^{4}} \frac{1-0.96 \exp (-0.0165 \mathrm{Bo})}{1-0.52 \exp (-0.0165 \mathrm{Bo})}} & \text { Bo }>4.55 \\
0 & \text { Bo } \leq 4.55
\end{array}\right.
$$

At low $\mathrm{N}_{\mathrm{f}}(<250)$ and $\mathrm{Bo}>4.55$, the correlation of White and Beardmore (1962) for $\Gamma$ (Eqns. 3 -4) is recommended. This low-Reynolds number large scale flow regime is never encountered for room-temperature air-water, but can be reached with more viscous fluids.

A blended capillary-to-large scale distribution parameter $\left(C_{0}\right)$ is proposed in this investigation. For the capillary contribution, the explicit correlation of Liu et al. (2005) is adopted.

$$
C_{0, \mathrm{Ca}}=\frac{1}{1-0.61 \mathrm{Ca}^{0.33}}
$$

For large scale flows, Bendiksen (1985) proposed a curve fit for $C_{0}$ (Eqn. 7) that decays from 2.29 (laminar, (Taylor, 1961)) at $\operatorname{Re}_{j}=36$, to 1.20 (turbulent, (Nicklin et al., 1962)) at $\operatorname{Re}_{j}=10^{6}$ (for Bo $\rightarrow \infty$ ). However, this curve fit diverges from these asymptotic limits outside of this range of $\operatorname{Re}_{j}$. A laminar counterpart (Eqn. 8) was also developed, but the laminar-to-turbulent transition was not addressed. In the present investigation, inferred laminar values of $C_{0}(>2)$ were found for $\operatorname{Re}_{j} \lesssim 800$, and fully converged turbulent values were found for $\operatorname{Re}_{j} \gtrsim 2500$ (Fig. 11).

Based on the above findings and considerations, the following large scale contribution is proposed. This transitional model reaches $99 \%$ of the laminar value of $C_{0}(2.29)$ at $\operatorname{Re}_{j}=312$, and $101 \%$ of the turbulent value (1.20) at $\mathrm{Re}_{j}=2500$.

$$
C_{0, \mathrm{LS}}=1.20+\frac{1.09}{1+\left(\operatorname{Re}_{j} / 805\right)^{4}}
$$




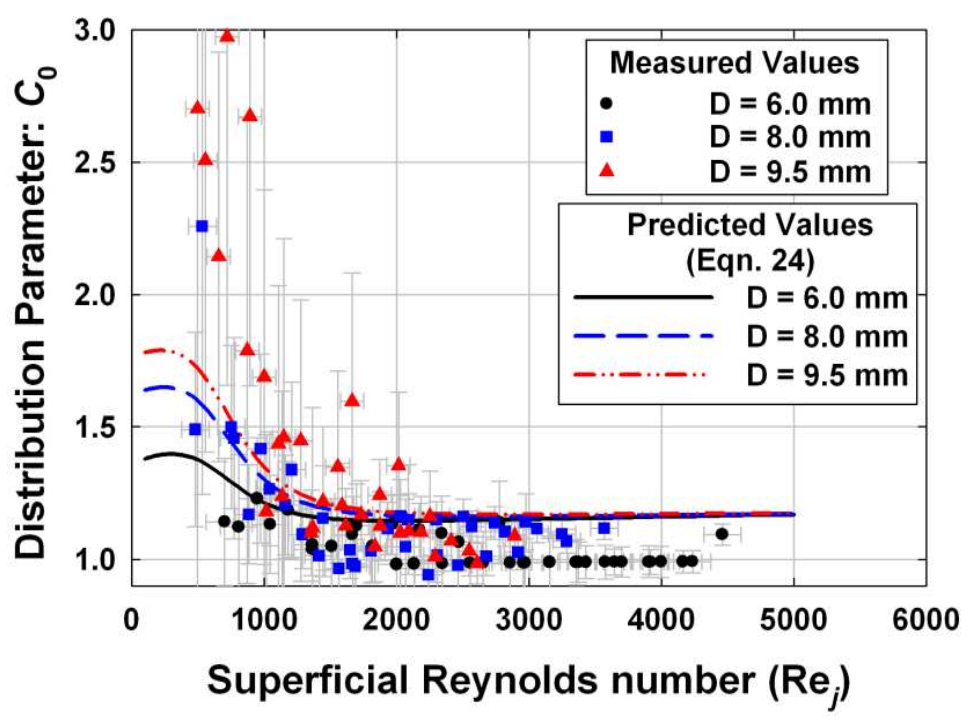

Figure 11 - Inferred $C_{0}$ from measurements (evaluating $\Gamma$ using Eqn. 20, assuming 25\% uncertainty) and predicted $C_{0}$ (Eqn. 24)

Bendiksen (1985) highlighted the competing effects of inertial (Re) and capillary (Bo) forces in his turbulent model for $C_{0}$. This approach is adopted here to blend the capillary and large scale contributions:

$$
\begin{aligned}
& f_{\mathrm{LS}}=\left(\frac{1}{1+4840 \mathrm{Re}_{j}^{-0.163}}\right)^{0.816 / \mathrm{Bo}} \\
& C_{0}=f_{\mathrm{LS}} C_{0, \mathrm{LS}}+\left(1-f_{\mathrm{LS}}\right) C_{0, \mathrm{Ca}}
\end{aligned}
$$

The large scale fraction $\left(f_{\mathrm{LS}}\right)$ blending factor was fit to yield approximately 0.3 for the $D=6.0$ mm cases, 0.5 for the $D=8.0 \mathrm{~mm}$ cases, and 0.6 for the $D=9.5 \mathrm{~mm}$ cases. These values were empirically found to deliver close agreement with experimental results for $U_{\mathrm{b}}$ from the present investigation. Future investigations over continuous ranges of Bo and wider ranges of $\mathrm{Re}_{j}$ could enable more rigorous fitting of this blending factor.

Predicted distribution parameter trends are presented for air-water flow in Fig. 11. At low $\operatorname{Re}_{j}$, 
measured values of $C_{0}$ for the 8.0 and $9.5 \mathrm{~mm}$ diameter tube flows exceed the established large scale laminar value of 2.29 . However, the propagated uncertainty is very large for these conditions, primarily due to the high uncertainty in the measured Taylor bubble rise velocities relative to the low superficial velocities. The proposed correlation for $C_{0}$ slightly exceeds measured values in the $6.0 \mathrm{~mm}$ diameter tube at high $\mathrm{Re}_{j}$. This trend is deemed acceptable due to the flow instability discussed in Section 3.1, which may skew measured velocities low. Further investigation into this phenomenon could enable improved prediction of bubble rise velocities for these lower Bond-number flows.

The performance of the overall proposed bubble rise velocity correlation is evaluated using:

- Intermediate scale results from the present investigation

- Capillary results from Liu et al. (2005) and Thulasidas et al. (1995)

- Large scale measurements for turbulent flow (Legius et al., 1997; van Hout et al., 2002) and with laminar-to-transitional flow (Collins et al., 1978).

Average absolute relative deviations $\left(\sum_{\mathrm{i}=1}^{n}\left|\left(U_{\mathrm{b} \text {,model }}-U_{\mathrm{b} \text {,experiment }}\right) / U_{\mathrm{b} \text {,experiment }}\right| / n\right)$ between pre-

Table 1 - Comparison of average absolute relative deviation in Taylor bubble rise velocity using proposed and previously published models

\begin{tabular}{lrrrr}
\hline Data set & $\begin{array}{c}\text { Proposed } \\
\text { Model }\end{array}$ & $\begin{array}{c}\text { Bendiksen } \\
(\mathbf{1 9 8 5})\end{array}$ & $\begin{array}{c}\text { de Cachard \& } \\
\text { Delhaye (1996) }\end{array}$ & $\begin{array}{c}\text { Liu et al. } \\
(\mathbf{2 0 0 5})\end{array}$ \\
\hline Present study & $10 \%$ & $22 \%$ & $15 \%$ & $19 \%$ \\
Liu et al. (2005) & $8 \%$ & $276 \%$ & $12 \%$ & $8 \%$ \\
Thulasidas et al (1995) & $12 \%$ & $255 \%$ & $15 \%$ & $12 \%$ \\
van Hout et al. (2002) & $13 \%$ & $12 \%$ & $14 \%$ & $35 \%$ \\
Legius et al. (1997) & $9 \%$ & $10 \%$ & $9 \%$ & $19 \%$ \\
Collins et al. (1978) & $4 \%$ & $3 \%$ & $7 \%$ & $74 \%$ \\
\hline
\end{tabular}


dicted and measured bubble translation velocities for these data sets are summarized in Table 1. Results from the proposed correlation are compared with those obtained using the models of Bendiksen (1985), de Cachard and Delhaye (1996), and Liu et al. (2005).

Based on these results, the proposed correlation achieves the best agreement among the models considered with experimentally measured bubble velocities in intermediate scale flows from the present investigation. Additionally, this model achieves comparable or better agreement than the other considered correlations for capillary and large scale flows. The correlation of Bendiksen (1985) yields excellent agreement with large scale flow results, but fails for capillary flows (Liu et al., 2005; Thulasidas et al., 1995). The model of de Cachard and Delhaye (1996) achieves good agreement in general, but assumes a fixed $C_{0}$ value of 1.2. Average errors are about $50 \%$ higher than with the proposed correlation for data sets where $C_{0}$ varied significantly (present study, (Collins et al., 1978; Liu et al., 2005)). The Liu et al. (2005) correlation is incorporated into the proposed model at the low-Bo limit, and achieves very good agreement at those scales. However, agreement is only fair for intermediate- and large-diameter tube flows. Capillary to large scale bubble rise velocity predictions using the proposed correlation are summarized in Fig. 12.

\subsection{Liquid-film thickness prediction}

Kinematic closure for a unit-cell based Taylor-flow model requires an additional parameter in addition to the bubble-rise velocity $\left(U_{\mathrm{b}}\right)$ and phase superficial velocities $\left(j_{\mathrm{L}}, j_{\mathrm{G}}\right)$. The continuity constraint requires that the average liquid slug velocity equals the total superficial velocity $\left(j=j_{\mathrm{L}}\right.$

$\left.+j_{\mathrm{G}}\right)$. Additionally, the net volumetric flow rate through cross-sections of a tube containing a liquid slug or a Taylor bubble and falling-film pair must be equal (Thulasidas et al., 1995).

$$
\alpha_{\mathrm{b}} U_{\mathrm{b}}-\left(1-\alpha_{\mathrm{b}}\right) U_{\mathrm{f}}=j
$$

Here, $\alpha_{\mathrm{b}}$ is the fraction of tube cross-section occupied by Taylor bubbles, and $U_{\mathrm{f}}$ is the average 


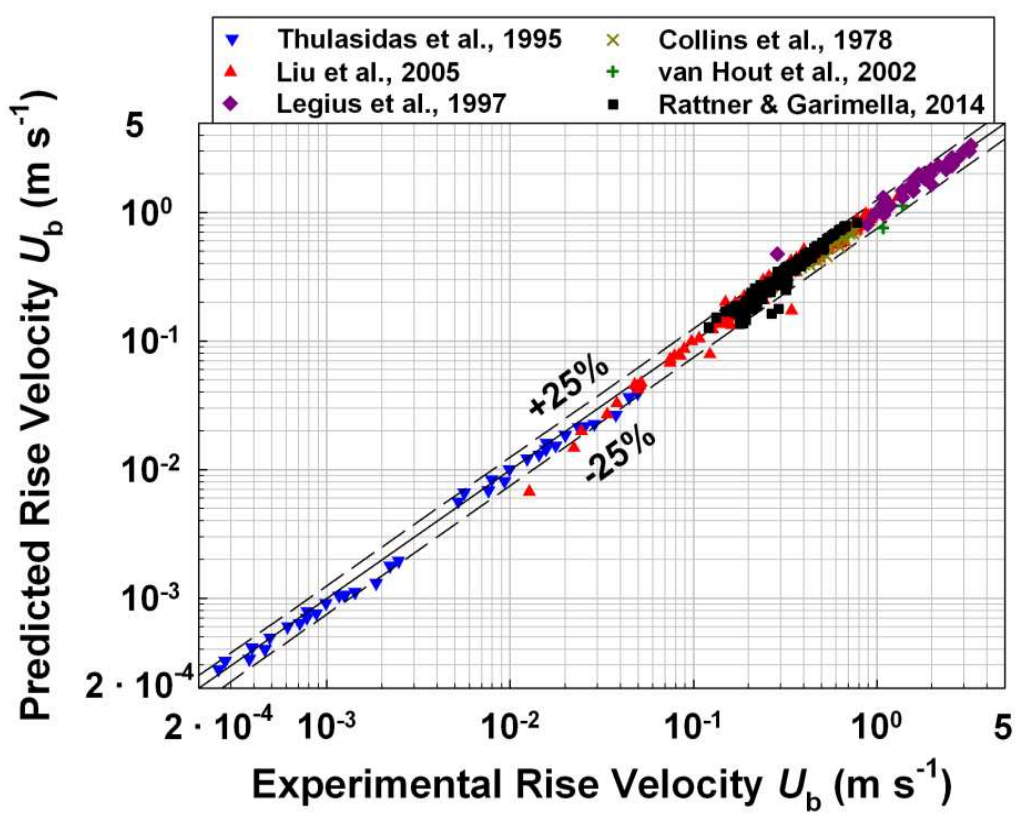

Figure 12 - Comparison of proposed Taylor bubble rise velocity model with measurements and values from the literature

liquid film velocity (positive downward). The average liquid film thickness around Taylor bubbles can be obtained as:

$$
\delta_{\mathrm{f}}=\frac{D}{2}\left(1-\alpha_{\mathrm{b}}^{1 / 2}\right)
$$

Based on these conservation equations and experimental results, $\operatorname{Re}_{\mathrm{f}}<180$ for all measured cases. This is well below the turbulent transition criterion of $\mathrm{Re}_{\mathrm{f}}>750$ suggested by de Cachard and Delhaye (1996); therefore, laminar falling films are assumed. The gas flow in rising bubbles is also assumed to be laminar because the bubble Reynolds number $\left(\operatorname{Re}_{\mathrm{b}}=\rho_{\mathrm{G}}\left(D-2 \delta_{\mathrm{f}}\right)\left(U_{\mathrm{b}}+U_{\mathrm{f}}\right) / \mu_{\mathrm{G}}\right)$ was found to be $<450$ for all measured cases.

Force balances were performed on the falling films accounting for bubble-to-film shear (assuming a laminar developing flow friction factor in the bubble), wall shear stress, and gravitational forces. For all considered cases, the bubble-to-film shear component was found to be insignifi- 
cant $\left(\tau_{\text {int }}<0.04 \mathrm{~N} \mathrm{~m}^{-2}, \tau_{\text {wall }}>1.61 \mathrm{~N} \mathrm{~m}^{-2}\right.$, and $\tau_{\text {int }} / \tau_{\text {wall }}<0.019$ for all cases $)$. Thus, the liquid film in intermediate scale Taylor flows can be assumed to travel down the tube wall with negligible interfacial shear. The laminar falling-film velocity result suggested by de Cachard and Delhaye (1996) (Eqn. 27) and Eqns. 25 - 26 (closure equations above) can thus be used to implicitly determine the film thickness in intermediate scale Taylor flows.

$$
U_{\mathrm{f}}=0.333 g \frac{\rho_{\mathrm{L}} \delta_{\mathrm{f}}^{2}}{\mu_{\mathrm{L}}}
$$

Predicted and measured film thicknesses (using correlated $U_{\mathrm{b}}$ from Section 4.1) from this investigation are compared in Fig. 13. An average absolute relative deviation $\left(\sum_{\mathrm{i}=1}^{n}\left|\left(\delta_{\mathrm{f}, \text { model }}-\delta_{\mathrm{f}, \text { experiment }}\right) / \delta_{\mathrm{f}, \text { experiment }}\right| / n\right)$ of $16 \%$ was found between measured and predicted values, and $86 \%$ of predictions were within $25 \%$ of measured values. Still, relatively wide scatter can be observed between predicted and measured values in Fig. 13. This can be attributed primarily to

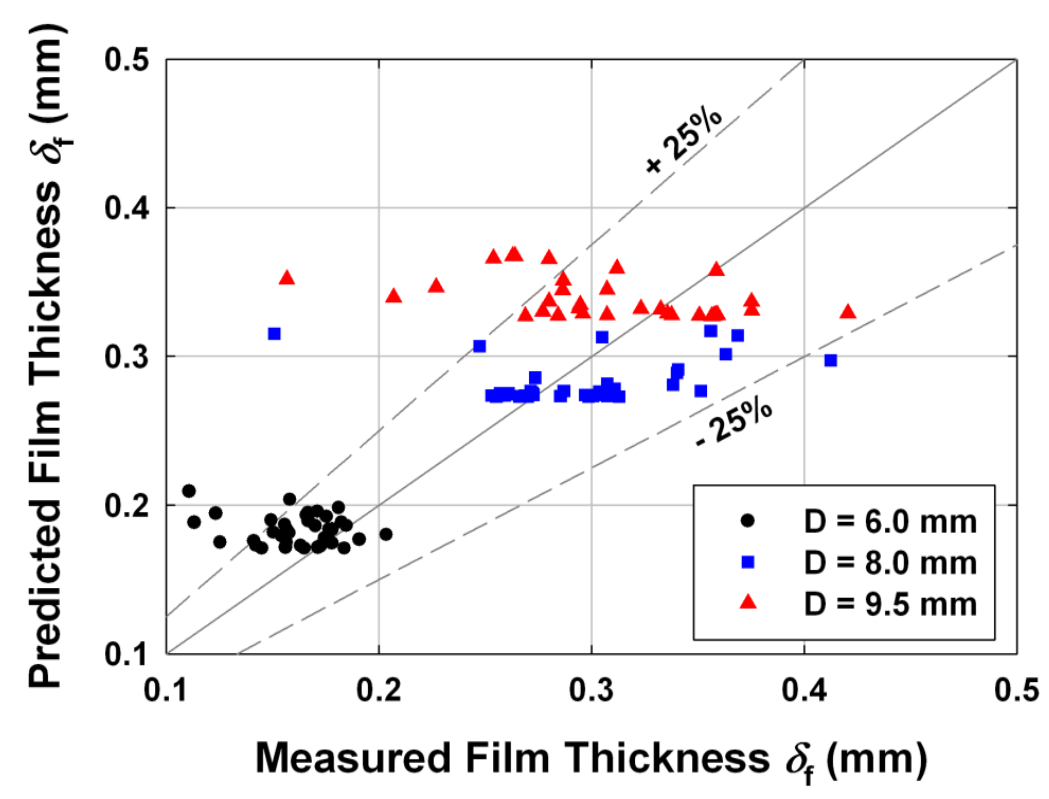

Figure 13 - Comparison of measured and predicted liquid-film thickness values 
the large experimental uncertainty in measuring film thicknesses typically $2-5$ pixels wide. Additionally, measured thicknesses may be skewed slightly low in cases where shading near the inner tube wall may be difficult to distinguish from the liquid-to-gas interface.

\subsection{Void fraction prediction}

The continuity constraint for gas-flow in a unit cell can be applied to determine the relative Taylor bubble length $\left(\beta=L_{\mathrm{b}} /\left(L_{\mathrm{b}}+L_{\mathrm{s}}\right)\right)$. If the liquid slug is not aerated, as was generally found to be the case for the studied intermediate scale Taylor flows, then:

$$
\beta=\frac{j_{\mathrm{G}}}{\alpha_{\mathrm{b}} U_{\mathrm{b}}}
$$

The overall void fraction can then be determined as:

$$
\alpha=\alpha_{\mathrm{b}} \beta
$$

This approach only requires knowledge of the superficial flow rates, the bubble rise velocity (correlated in Section 4.1), and the liquid film thickness (modeled in Section 4.2). Additionally, this method automatically satisfies the continuity constraint for the simplified unit-cell model, and is therefore preferable to application of a "general purpose" void fraction correlation. Predicted and measured void fraction values from this study are presented in Fig. 14. In these results, $U_{\mathrm{b}}$ and $\delta_{\mathrm{f}}$ are evaluated following the approaches presented in Sections 4.1 and 4.2, respectively. An average deviation of $9 \%$ in void fraction is seen, and $95 \%$ of data points are predicted within $25 \%$ of the measured values. 


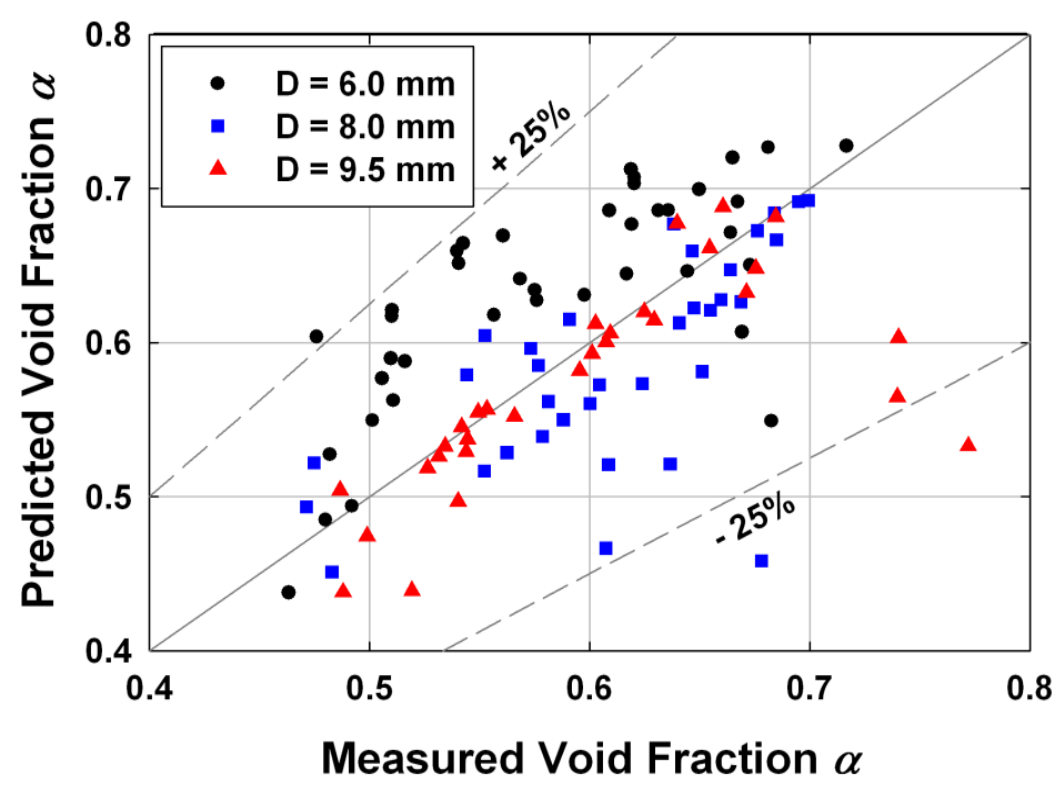

Figure $14-$ Comparison of measured and predicted void fraction values

\subsection{Taylor bubble and liquid slug length prediction}

Taylor bubble and liquid slug length values are primarily employed in models for predicting the frequency of transitional pressure drops between bubble and slug segments. For large scale flows, the average relative liquid slug length $\left(L_{\mathrm{s}} / D\right)$ has been reported to be constant and universal, but widely varying values $(6-48)$ have been published. This substantial difference between the results from different investigations has not proved to be a significant issue in large scale flow models because bubbles and slugs are typically many tube diameters in length; thus, fully developed frictional pressure gradients dominate transitional pressure drops (Sylvester, 1987). In contrast, average liquid slug lengths of $1-6 D$ were seen in the present study for intermediate tubediameter flows. As such, the relative effect of transitional pressure drops can be expected to be much greater in these cases, and more accurate prediction of segment lengths is warranted.

In this study, video data were obtained approximately $500-600 \mathrm{~mm}(55-100 \mathrm{D})$ above the inlet ports. It is thus possible that the short measured slugs and bubbles could be due to entrance ef- 
fects. However, there are no clear trends of measured slug lengths varying with Reynolds number $\left(\mathrm{Re}_{j}\right)$ as might be expected for entrance effects. Additionally, measured relative slug lengths $\left(L_{\mathrm{s}} / D\right)$ are actually greater in the larger diameter tubes where measurements are collected relatively closer to the inlets (in dimensionless length: $x / D$ ). Future investigations with longer test sections could help verify whether these short segment lengths persist further downstream.

In the present investigation, Taylor bubbles were generally found to be much longer than liquid slugs $\left(L_{\mathrm{b}} / D \sim 2-18\right)$. Therefore, for known $\beta$, more accurate predictions of unit-cell length and frequency of transitional pressure drops can be obtained by correlating $L_{\mathrm{b}}$ rather than $L_{\mathrm{s}}$. Additionally, trends of $L_{\mathrm{b}} / D$ with flow rate were more apparent than for $L_{\mathrm{s}}$.

Laborie et al. (1999) proposed a capillary-scale power-law correlation for $L_{\mathrm{b}} / D$ in terms of Bond number and a bubble Reynolds number (Eqn. 16). However, this approach might not apply at other scales where similar relative bubble lengths are observed, but Re and Bo vary by orders of magnitude. Kreutzer et al. (2003) proposed a correlation for slug length in terms of void fraction (Eqn. 17). This approach holds promise at all scales because relative bubble and slug lengths and void fractions are of similar order for Taylor flow at all length scales.

Here, a correlation for relative Taylor bubble length in intermediate scale flows is proposed based on image analysis results from Section 3.4.

$$
\frac{L_{\mathrm{b}}}{D}=1.813\left\{\exp \left[1.815-0.866\left(\frac{U_{\mathrm{b}}}{j}\right)^{2.176}\right]\left(\frac{\alpha}{1-\alpha}\right)^{1.637}+1\right\}
$$

This correlation yields a minimum bubble length of $L_{\mathrm{b}} / D=1.813$, and thus predicts elongated $\left(L_{\mathrm{b}} / D>1\right)$ bubbles for all cases of Taylor flow. Additionally, predicted bubble lengths scale to $\infty$ as $\alpha \rightarrow 1$. As such, very long bubbles are predicted at the Taylor flow-to-annular flow transition. 
In the large Bond number limit, this correlation converges to $L_{\mathrm{b}} / D=1.813$, which is generally smaller than the first order approximation (assuming $\alpha_{\mathrm{b}}=1, L_{\mathrm{s}} / D=16$ ):

$$
\left(\frac{L_{\mathrm{b}}}{D}\right)_{\mathrm{Bo} \rightarrow \infty} \approx \frac{16}{U_{\mathrm{b}} / j_{\mathrm{G}}-1}
$$

In future models, this limit could be incorporated, perhaps with a Bond-number based blending between intermediate and large scale flow bubble length predictions. At present, there is insufficient information about the transition from the intermediate- to large scale regimes to develop such a correlation.

A comparison of the performance of bubble/slug-length models is presented in Table 2. The considered models include:

- The proposed correlation (Eqn. 30)

- The capillary scale correlation of Laborie et al. (1999) (Eqn. 16)

- The capillary scale correlation of Kreutzer et al. (2003) (Eqn. 17)

- The large scale (high Bo) result of Taitel et al. (1980) $\left(L_{\mathrm{s}} / D=16\right)$

These models are evaluated with experimental data, including:

- Measurements from this study - closure for $U_{\mathrm{b}}, \delta_{\mathrm{f}}, \alpha, \beta$ obtained using models from the preceding sections)

- Large scale results from Pinto et al. (2001) - $U_{\mathrm{b}}, L_{\mathrm{s}}, L_{\mathrm{b}}$ available from experimental data, we assume $\alpha \sim \beta$

- Capillary data from Shao et al. (2011) - $U_{\mathrm{b}}$ estimated using Liu et al. (2005) correlation (Eqn. 10), $\delta_{\mathrm{f}}$ estimated using Fairbrother and Stubbs (1935) correlation (Eqn. 13), $\alpha$ and $\beta$ obtained from continuity equations 
Table 2 - Comparison of average absolute deviation (and order of magnitude error) in Taylor bubble lengths using proposed and previously published models

\begin{tabular}{lcccc}
\hline Data set & $\begin{array}{c}\text { Proposed } \\
\text { Model }\end{array}$ & $\begin{array}{c}\text { Laborie et al. } \\
(\mathbf{1 9 9 9})\end{array}$ & $\begin{array}{c}\text { Kreutzer et al. } \\
\mathbf{( 2 0 0 3 )}\end{array}$ & $\begin{array}{c}\text { Taitel et al. } \\
(\mathbf{1 9 8 0})\end{array}$ \\
\hline Present study & $22 \%(0.11)$ & $85 \%(0.87)$ & $44 \%(0.27)$ & $453 \%(0.70)$ \\
Pinto et al. (2001) & $73 \%(0.58)$ & $99 \%(2.06)$ & $83 \%(0.78)$ & $12 \%(0.05)$ \\
Shao et al (2011) & $140 \%(0.45)$ & $1748 \%(0.99)$ & $58 \%(0.45)$ & $437 \%(0.61)$ \\
\hline
\end{tabular}

Average absolute deviations (AAD) from experimental values are summarized in Table 2. Average logarithmic-scale errors are also reported in parentheses $\left(\left|\log _{10}\left(L_{\mathrm{b} \text {,experiment }} / L_{\mathrm{b}, \text { model }}\right)\right|\right)$ to indicate prediction errors in terms of orders of magnitude.

Based on these results, there are no generally valid Taylor bubble length models available in the literature. The proposed correlation achieves good agreement with the present data $(22 \%$ AAD), and predicts capillary and large scale results from the literature to within approximately 0.5 orders of magnitude. The Kreutzer et al. (2003) correlation achieves reasonable accuracy for the present data set (44\% AAD), and similar logarithmic-scale accuracy to the present correlation at other scales. The Taitel et al. (1980) result is very accurate (12\% AAD) for the large scale data of Pinto et al. (2001), but significantly over-predicts bubble lengths at the capillary and intermediate scales.

Using the proposed correlation, $66 \%$ of average bubble lengths from the present study are predicted within $25 \%$ of the measured values (91\% within $50 \%)$. Slug lengths are also predicted using this correlation and closure models described in the previous sections. Only $17 \%$ of average slug length values are predicted within $25 \%$ of measured values $(\mathrm{AAD}=74 \%, 52 \%$ within $50 \%)$. This limited agreement is unsurprising considering the wide range of average slug lengths reported in the large scale flow literature. Taylor bubbles were generally found to be much longer 

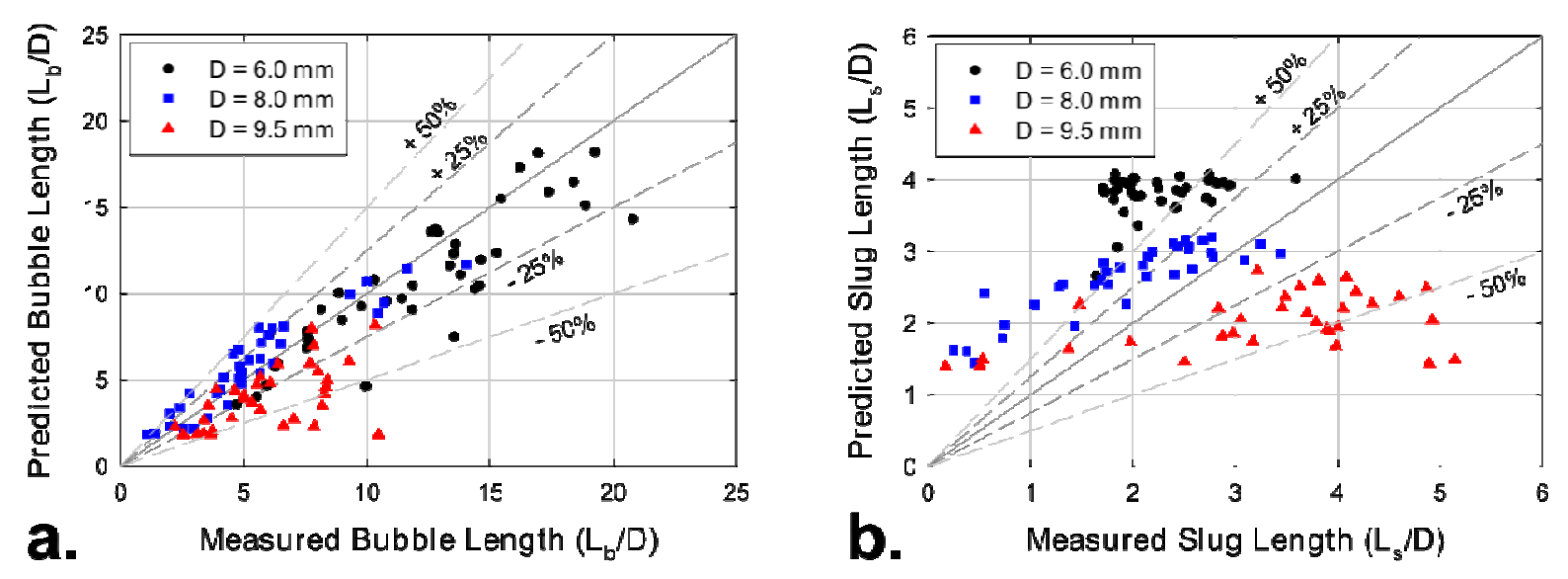

Figure 15 - Comparison of measured and predicted (a) Taylor bubble lengths, and (b) liquid slug lengths

than liquid slugs in the present study; measured values of $\beta$ ranged from $0.6 \lesssim \beta \lesssim 0.9$. Thus, unit cell lengths, and frequency of transition pressure drops, should be predicted reasonably well even with large uncertainty in $L_{\mathrm{s}}$. Predicted and average measured bubble and slug lengths from this investigation are presented in Fig. 15.

\section{DISCUSSION}

This investigation demonstrated experimentally that intermediate scale Taylor flows $(5 \lesssim$ Bo $\lesssim 40)$ are significantly different from those observed at the capillary and large scales. In particular, Taylor bubble rise velocity and bubble and slug length trends differ from those found at other scales. New correlations have been proposed to predict intermediate scale bubble rise velocity (also validated at other scales) and average Taylor bubble length. Laminar-falling film results were demonstrated to apply for the liquid-films around Taylor bubbles at this scale. Closure models based on conservation laws and the simplified unit-cell model were employed to predict $\alpha$, $\beta$, and $L_{\mathrm{s}}$. This study thus provides sufficient information for kinematic closure of Taylor flows. 
Pressure drop information for the liquid slugs, bubble regions, and bubble-to-slug transitions is required for dynamic closure, and is a critical topic for future investigation.

One open question raised in this investigation is the effective entry length for intermediate scale Taylor flows. This is particularly relevant for measurement and prediction of bubble and slug lengths. Average bubble and slug lengths increase in the flow direction, up to stable values, as trailing bubbles merge with leading ones (Dukler et al., 1985). It is possible therefore that short measured liquid slugs had not reached fully developed lengths. Taitel et al. (1980) proposed the following entrance length correlation for slug flow:

$$
\frac{L_{\mathrm{ent}}}{D}=40.6\left(\frac{j}{\sqrt{g D}}+0.22\right)
$$

In this study, the camera recording window $(\sim 500-600 \mathrm{~mm}$ above the inlet) was above this point except for some of the highest flow rate $D=6.0$ and $8.0 \mathrm{~mm}$ cases. However, this entrance length model was developed for large scale turbulent flows, and may not apply for intermediate scale conditions. Future investigations focusing on Taylor-flow entrance lengths are therefore warranted.

In this investigation, a capillary-to-large scale blending model was proposed for predicting Taylor bubble rise velocity (Eqn. 23). A similar approach could assist in developing a universal Taylor bubble length model. However, insufficient data were available in the literature to fit such a blending model at this point. Additionally, the blending model for Taylor bubble velocity was fit empirically, because measurements from the present study were only collected for three distinct values of Bo. Future investigations focusing on the transition from the capillary-to-large scale limits would thus be of great value. The governing regime scale is primarily a function of Bond 
number $\left(\mathrm{Bo}=\left(\rho_{\mathrm{L}}-\rho_{\mathrm{G}}\right) g D^{2} / \sigma\right)$, which is only a function of tube diameter and fluid properties. Thus, it is more difficult to perform experiments over a continuous range of Bond numbers than other parameters, which can be varied simply with flow rate. However, this is an attractive avenue for simulation-based studies.

\section{CONCLUSIONS}

In this investigation, experimental air-water studies were performed for vertical upward intermediate scale Taylor flows. High speed photography was employed to enable measurement of flow parameters including Taylor bubble velocity, liquid film thickness, void fraction, and bubble and slug length. These measured parameters are summarized for reference in Appendix A.

Correlations were developed for Taylor bubble rise velocities at all scales, and for Taylor bubble lengths in intermediate scale flows. Laminar falling-film results were demonstrated to apply for liquid films around Taylor bubbles in intermediate scale flows. Additionally, the continuity constraint and simplified unit-cell model were employed to predict void fraction and average liquid slug length. This study thus provides models for kinematic closure of intermediate scale Taylor flows. Development of dynamic closure models (for pressure drop) is a key area of interest for future studies, and will enable comprehensive Taylor-flow models at this scale.

This investigation demonstrated that intermediate scale Taylor flows $(5 \lesssim$ Bo $\lesssim 40)$ are fundamentally different from those found at the capillary and large scales. Additional classes of forces and phenomena must be considered as compared with flows at either limit. This study identified a number of open topics for future Taylor flow investigations including the nature of the strong flow intermittency measured at Bo $=4.9$ (Section 3.1), the trends of bubble and liquid slug lengths in the transition from capillary to large scale flows (Section 4.4), and prediction of entrance lengths for intermediate scale flows. 
Rattner and Garimella, 2014

\section{ACKNOWLEDGEMENTS}

The authors wish to acknowledge generous financial support from the U.S. Department of Energy through the Krell Institute (contract DE-FG02-97ER25308).

\section{ROLE OF FUNDING SOURCE}

This project was sponsored by the Krell Institute through U.S. Department of Energy contract DE-FG02-97ER25308. The funding agencies were not materially involved in the research or reporting efforts. 


\section{REFERENCES}

Anderson, R., Zhang, L., Ding, Y., Blanco, M., Bi, X., Wilkinson, D.P., 2010. A critical review of two-phase flow in gas flow channels of proton exchange membrane fuel cells, Journal of Power Sources 195, 4531-4553.

Angeli, P., Gavriilidis, A., 2008. Hydrodynamics of Taylor flow in small channels: A Review, Proceedings of the Institution of Mechanical Engineers, Part C: Journal of Mechanical Engineering Science 222, 737-751.

Argyropoulos, P., Scott, K., Taama, W.M., 1999. Carbon dioxide evolution patterns in direct methanol fuel cells, Electrochimica Acta 44, 3575-3584.

Barnea, D., Brauner, N., 1985. Holdup of the liquid slug in two phase intermittent flow, International Journal of Multiphase Flow 11, 43-49.

Barnea, D., Taitel, Y., 1993. A model for slug length distribution in gas-liquid slug flow, International Journal of Multiphase Flow 19, 829-838.

Bendiksen, K.H., 1985. On the motion of long bubbles in vertical tubes, International Journal of Multiphase Flow 11, 797-812.

Bretherton, F.P., 1961. The motion of long bubbles in tubes, Journal of Fluid Mechanics 10, 166188.

Brill, J.P., Schmidt, Z., Coberly, W.A., Herring, J.D., Moore, D.W., 1981. Analysis of two phase tests in large diameter flow lines in Prudhoe bay field, Society of Petrochemical Engineering Journal 271, 363-378.

Brotz, W., 1952. Uber die Vorausberedinung der Absorptions geschwineig von Gayen instromenden flussig kectsschichten, Chem. Ing. Tech. 26, 470-478.

Bugg, J.D., Saad, G.A., 2002. The velocity field around a Taylor bubble rising in a stagnant viscous fluid: numerical and experimental results, International Journal of Multiphase Flow 28, 791-803.

Collins, R., Moraes, F.F.D., Davidson, J.F., Harrison, D., 1978. The motion of a large gas bubble rising through liquid flowing in a tube, Journal of Fluid Mechanics 89, 497-514.

Dassault Systems, 2013. PhotoView 360, Waltham, MA.

Davies, R.M., Taylor, G.I., 1950. The mechanics of large bubbles rising through extended liquids and through liquids in tubes, Proceedings of the Royal Society A 200, 375-390.

de Cachard, F., Delhaye, J.M., 1996. A slug-churn flow model for small-diameter airlift pumps, International Journal of Multiphase Flow 22, 627-649.

Delano, A.D., 1998. Design and analysis of the Einstein refrigeration cycle, PhD Thesis, Georgia Institute of Technology, Atlanta, GA.

Dukler, A.E., Maron, D.M., Brauner, N., 1985. A physical model for predicting the minimum stable slug length, Chemical Engineering Science 40, 1379-1385.

Dumitrescu, D.T., 1943. Strömung an einer Luftblase im senkrechten Rohr, Zeitschrift für Angewandte Mathematik und Mechanik 23, 139-149. 
Fairbrother, F., Stubbs, A.E., 1935. Studies in electro-endosmosis. Part VI. The "Bubble-tube" method of measurement, Journal of the Chemical Society, 527-529.

Fernandes, R.C., 1981. Experimental and theoretical studies of isothermal upward gas-liquid flows in vertical tubes, PhD Thesis, University of Houston, Houston, TX.

Fernandes, R.C., Semiat, R., Dukler, A.E., 1983. Hydrodynamic model for gas-liquid slug flow in vertical tubes, AIChE Journal 29, 981-989.

Funada, T., Joseph, D.D., Maehara, T., Yamashita, S., 2005. Ellipsoidal model of the rise of a Taylor bubble in a round tube, International Journal of Multiphase Flow 31, 473-491.

Gao, Z.K., Jin, N.D., 2012. Characterization of chaotic dynamic behavior in the gas-liquid slug flow using directed weighted complex network analysis, Physica A: Statistical Mechanics and its Applications 391, 3005-3016.

Garimella, S., 2000. Microchannel components for absorption space-conditioning systems ASHRAE Transactions Symposia 106, 453-462.

Garimella, S., 2004. Condensation flow mechanisms in microchannels: basis for pressure drop and heat transfer models, Heat Transfer Engineering 25, 104-116.

Hussaini, I.S., Wang, C.Y., 2009. Visualization and quantification of cathode channel flooding in PEM fuel cells, Journal of Power Sources 187, 444-451.

Kawahara, A., Chung, P.M.Y., Kawaji, M., 2002. Investigation of two-phase flow pattern, void fraction and pressure drop in a microchannel, International Journal of Multiphase Flow 28, 1411-1435.

Khandekar, S., Panigrahi, P.K., Lefèvre, F., Bonjour, J., 2010. Local hydrodynamics of flow in a pulsating heat pipe: A review, Frontiers in Heat Pipes 1.

Kreutzer, M.T., Wei, W., Kapteijn, F., Moulijn, J.A., Heiszwolf, J.J., 2003. Pressure drop of Taylor flow in capillaries: impact of slug length, International Conference on Microchannels and Minichannels, Rochester, New York.

Laborie, S., Cabassud, C., Durand-Boulier, L., Laine, J.M., 1999. Characterisation of gas-liquid two-phase flow inside capillaries, Chemical Engineering Science 54, 5723-5735.

Legius, H.J.W.M., Akker, H.E.A.v.d., Narumo, T., 1997. Measurements on wave propagation and bubble and slug velocities in cocurrent upward two-phase flow, Experimental Thermal and Fluid Science 15, 267-278.

Liu, H., Vandu, C.O., Krishna, R., 2005. Hydrodynamics of Taylor flow in vertical capillaries: flow regims, bubble rise velocity, liquid slug length, and pressure drop, Industrial \& Engineering Chemistry Research 44, 4884-4897.

Llewellin, E.W., Del Bello, E., Taddeucci, J., Scarlato, P., Lane, S.J., 2011. The thickness of the falling film of liquid around a Taylor bubble, Proceedings of the Royal Society A.

Mandal, T.K., Das, G., Das, P.K., 2007. Prediction of rise velocity of a liquid Taylor bubble in a vertical tube, Physics of Fluids 19, 2007.

Moissis, R., Griffith, P., 1962. Entrance effects in two-phase slug flow, Journal of Heat Transfer 84, 29-39. 
Nguen, K., Daw, C.S., Chakka, P., Cheng, M., Bruns, D.D., Finney, C.E.A., Kennell, M.B., 1996. Spatio-temporal dynamics in a train of rising bubbles, The Chemical Engineering Journal and the Biochemical Engineering Journal 64, 191-197.

Nicklin, D.J., Wilkes, J.O., Davidson, J.F., 1962. Two-phase flow in vertical tubes, Transactions of the Institution of Chemical Engineers 40, 61-68.

Nogueira, S., Riethmuler, M.L., Campos, J.B.L.M., Pinto, A.M.F.R., 2006a. Flow in the nose region and annular film around a Taylor bubble rising through vertical columns of stagnant and flowing Newtonian liquids, Chemical Engineering Science 61, 845 - 857.

Nogueira, S., Riethmuler, M.L., Campos, J.B.L.M., Pinto, A.M.F.R., 2006b. Flow patterns in the wake of a Taylor bubble rising through vertical columns of stagnant and flowing Newtonian liquids: An experimental study, Chemical Engineering Science 61, 7199-7212.

Pinto, A.M.F.R., Coelho-Pinheiro, M.N., Campos, J.B.L., 2001. On the interaction of Taylor bubbles rising in two-phase co-current slug flow in vertical columns: turbulent wakes, Experiments in Fluids 31, 643-652.

Reinemann, D.J., Parlange, J.Y., Timmons, M.B., 1990. Theory of small-diameter airlift pumps, International Journal of Multiphase Flow 16, 113-122.

Schmidt, Z., 1977. Experimental study of two-phase slug flow in a pipeline-riser pipe system, $\mathrm{PhD}$ Thesis, University of Tulsa, Tulsa, AZ.

Shao, N., Gavriilidis, A., Angeli, P., 2011. Effect of inlet conditions on Taylor bubble length in microchannels, Heat Transfer Engineering 32, 1117-1125.

Sylvester, N.D., 1987. A mechanistic model for two-phase vertical slug flow in pipes, Journal of Energy Resources Technology 109, 206-213.

Taitel, Y., Barnea, D., 1990. Two-phase slug flow, Advances in Heat Transfer 20, 83-132.

Taitel, Y., Barnea, D., Dukler, A.E., 1980. Modelling flow pattern transitions for steady upward gas-liquid flow in vertical tubes, AIChE Journal 26, 345-354.

Taylor, G.I., 1961. Deposition of a viscous fluid on the wall of a tube, Journal of Fluid Mechanics $10,161-165$.

Thulasidas, T.C., Abraham, M.A., Cerro, R.L., 1995. Bubble-train flow in capillaries of circular and square cross section, Chemical Engineering Science 50, 183-199.

Triplett, K.A., Ghiaasiaan, S.M., Abdel-Khalik, S.I., LeMouel, A., McCord, B.N., 1999. Gasliquid two-phase flow in microchannels: Part II: void fraction and pressure drop, International Journal of Multiphase Flow 25, 395-410.

van Hout, R., Barnea, D., Shemer, L., 2002. Translational velocities of elongated bubbles in continuous slug flow, International Journal of Multiphase Flow 28, 1333-1350.

Viana, F., Pardo, R., Yánez, R., Trallero, J.L., Joseph, D.D., 2003. Universal correlation for the rise velocity of long gas bubbles in round pipes, Journal of Fluid Mechanics 494, 379-398.

von Platen, B.C., Munters, C.G., 1928. Refrigerator, Patent 1,685,764.

Wang, S., Zhang, H., Wang, J., 2009. Cryogenic liquid slug and Taylor bubble length distributions in an inclined tube, Chinese Journal of Chemical Engineering 17, 20-26. 
White, E.T., Beardmore, R.H., 1962. The rise velocity of single cylindrical air bubbles through liquids contained in vertical tubes, Chemical Engineering Science 17, 351-361.

Winkler, J., Killion, J., Garimella, S., 2012. Void fractions for condensing refrigerant flow in small channels. Part II: Void fraction measurement and modeling, International Journal of Refrigeration 35, 246-262.

Woldesemayat, M.A., Ghajar, A.J., 2007. Comparison of void fraction correlations for different flow patterns in horizontal and upward inclined pipes, International Journal of Multiphase Flow 33, 347-370. 
APPENDIX A: EXPERIMENTAL MEASUREMENTS

\begin{tabular}{|c|c|c|c|c|c|c|c|c|c|}
\hline \multicolumn{10}{|c|}{$6.0 \mathrm{~mm}$ diameter tube, $832 \mathrm{~mm}$ long } \\
\hline $\begin{array}{c}S_{r} \\
( \pm \mathbf{0 . 0 3})\end{array}$ & $\begin{array}{c}\nabla P \\
\left(\mathbf{P a ~ m}^{-1}\right) \\
( \pm 260)\end{array}$ & $\begin{array}{c}\mathbf{V}_{\mathbf{G}} \\
\left(\mathbf{m l ~ \text { min} ^ { - 1 }}\right) \\
( \pm \mathbf{3 8})\end{array}$ & $\begin{array}{c}V_{L} \\
\left(\mathrm{ml} \mathrm{min}^{-1}\right)\end{array}$ & $\begin{array}{c}\mathbf{U}_{\mathrm{b}} \\
\left(\mathrm{m} \mathrm{s}^{-1}\right)\end{array}$ & 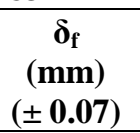 & $\begin{array}{c}\alpha \\
( \pm 0.03)\end{array}$ & $\beta$ & $\begin{array}{c}L_{\mathrm{b}} \\
(\mathbf{m m})\end{array}$ & $\begin{array}{c}L_{\mathrm{s}} \\
(\mathbf{m m})\end{array}$ \\
\hline 0.30 & 2940 & 142 & $47.7 \pm 0.3$ & $0.13 \pm 0.01$ & 0.11 & 0.68 & 0.893 & $60 \pm 19$ & $9.8 \pm 1.9$ \\
\hline 0.30 & 2940 & 212 & $70.1 \pm 0.5$ & $0.19 \pm 0.02$ & 0.11 & 0.67 & 0.904 & $81 \pm 24$ & $12.3 \pm 3.3$ \\
\hline 0.30 & 2940 & 283 & $85.4 \pm 0.6$ & $0.24 \pm 0.02$ & 0.13 & 0.64 & 0.880 & $86 \pm 27$ & $14.9 \pm 3.3$ \\
\hline 0.30 & 2940 & 354 & $96.3 \pm 0.6$ & $0.30 \pm 0.03$ & 0.15 & 0.66 & 0.918 & $92 \pm 27$ & $12.1 \pm 2.7$ \\
\hline 0.30 & 2940 & 425 & $107.2 \pm 0.7$ & $0.36 \pm 0.03$ & 0.16 & 0.63 & 0.914 & $77 \pm 24$ & $11.0 \pm 2.0$ \\
\hline 0.30 & 2940 & 472 & $115.1 \pm 0.8$ & $0.39 \pm 0.02$ & 0.14 & 0.67 & 0.918 & $125 \pm 32$ & $16.5 \pm 3.7$ \\
\hline 0.30 & 2940 & 543 & $125.0 \pm 0.9$ & $0.43 \pm 0.03$ & 0.14 & 0.65 & 0.917 & $113 \pm 29$ & $14.7 \pm 3.4$ \\
\hline 0.30 & 2940 & 614 & $136.7 \pm 1.0$ & $0.51 \pm 0.06$ & 0.15 & 0.62 & 0.890 & $93 \pm 29$ & $16.4 \pm 4.4$ \\
\hline 0.30 & 2940 & 661 & $142.0 \pm 1.2$ & $0.46 \pm 0.04$ & 0.15 & 0.62 & 0.899 & $104 \pm 26$ & $17.3 \pm 3.6$ \\
\hline 0.30 & 2940 & 708 & $145.0 \pm 1.2$ & $0.45 \pm 0.04$ & 0.16 & 0.62 & 0.895 & $110 \pm 27$ & $17.6 \pm 3.6$ \\
\hline 0.30 & 2940 & 779 & $148.5 \pm 1.3$ & $0.49 \pm 0.03$ & 0.16 & 0.67 & 0.925 & $97 \pm 22$ & $11.9 \pm 2.1$ \\
\hline 0.30 & 2940 & 850 & $151.0 \pm 1.3$ & $0.50 \pm 0.03$ & 0.15 & 0.68 & 0.932 & $102 \pm 23$ & $12.2 \pm 1.8$ \\
\hline 0.30 & 2940 & 920 & $160.2 \pm 1.4$ & $0.59 \pm 0.03$ & 0.17 & 0.72 & 0.947 & $116 \pm 24$ & $13.7 \pm 2.4$ \\
\hline 0.40 & 3920 & 142 & $76.4 \pm 0.4$ & $0.15 \pm 0.03$ & 0.16 & 0.49 & 0.757 & $33 \pm 20$ & $11.1 \pm 2.1$ \\
\hline 0.40 & 3920 & 212 & $106.5 \pm 0.7$ & $0.23 \pm 0.02$ & 0.16 & 0.50 & 0.736 & \pm 15 & $14.5 \pm 3.5$ \\
\hline 0.40 & 3920 & 283 & $124.5 \pm 0.8$ & $0.26 \pm 0.04$ & 0.17 & 0.51 & 0.809 & $37 \pm 15$ & $10.2 \pm 1.3$ \\
\hline 0.40 & 3920 & 354 & $137.1 \pm 0.9$ & $0.31 \pm 0.03$ & 0.18 & 0.56 & 0.867 & $9 \pm 14$ & $10.9 \pm 1.9$ \\
\hline 0.40 & 3920 & 425 & $151.3 \pm 1.0$ & $0.32 \pm 0.04$ & 0.16 & 0.58 & 0.844 & $3 \pm 22$ & $12.0 \pm 2.5$ \\
\hline 0.40 & 3920 & 472 & $161.2 \pm 1.0$ & $0.42 \pm 0.05$ & 0.18 & 0.57 & 0.840 & $7 \pm 25$ & $21.6 \pm 4.5$ \\
\hline 0.40 & 3920 & 543 & $173.9 \pm 1.3$ & $0.40 \pm 0.05$ & 0.17 & 0.54 & 0.854 & \pm 22 & $16.7 \pm 2.7$ \\
\hline 0.40 & 3920 & 614 & $185.9 \pm 1.3$ & $0.45 \pm 0.05$ & 0.18 & 0.54 & 0.839 & \pm 27 & $17.0 \pm 2.8$ \\
\hline 0.40 & 3920 & 661 & $193.0 \pm 1.4$ & $0.48 \pm 0.05$ & 0.18 & 0.54 & 0.857 & \pm 25 & $17.6 \pm 3.2$ \\
\hline 0.40 & 3920 & 708 & $199.1 \pm 1.7$ & $0.45 \pm 0.04$ & 0.17 & 0.56 & 0.882 & 21 & $13.5 \pm 2.0$ \\
\hline 0.40 & 3920 & 779 & $206.6 \pm 1.7$ & $0.49 \pm 0.02$ & 0.17 & 0.62 & 0.916 & 16 & $10.9 \pm 1.3$ \\
\hline 0.40 & 3920 & 850 & $209.6 \pm 1.8$ & $0.52 \pm 0.02$ & 0.18 & 0.61 & 0.894 & $6 \pm 17$ & $12.1 \pm 2.0$ \\
\hline 0.40 & 3920 & 920 & $224.4 \pm 1.9$ & $0.60 \pm 0.02$ & 0.17 & 0.64 & 0.919 & $7 \pm 17$ & $10.9 \pm 2.2$ \\
\hline 0.50 & 4900 & 142 & $114.4 \pm 0.7$ & $0.19 \pm 0.02$ & 0.12 & 0.46 & 0.724 & \pm 18 & $11.5 \pm 1.4$ \\
\hline 0.50 & 4900 & 212 & $155.8 \pm 1.0$ & $0.23 \pm 0.03$ & 0.16 & 0.48 & 0.783 & $36 \pm 15$ & $11.1 \pm 1.3$ \\
\hline 0.50 & 4900 & 283 & $175.6 \pm 1.3$ & $0.31 \pm 0.04$ & 0.17 & 0.48 & 0.765 & $38 \pm 19$ & $11.9 \pm 1.9$ \\
\hline 0.50 & 4900 & 354 & $186.7 \pm 1.3$ & $0.29 \pm 0.03$ & 0.17 & 0.51 & 0.815 & $45 \pm 18$ & $11.3 \pm 1.4$ \\
\hline 0.50 & 4900 & 425 & $209.1 \pm 1.8$ & $0.32 \pm 0.03$ & 0.18 & 0.51 & 0.802 & $46 \pm 16$ & $13.4 \pm 2.5$ \\
\hline 0.50 & 4900 & 472 & $218.8 \pm 1.8$ & $0.38 \pm 0.04$ & 0.19 & 0.52 & 0.807 & $46 \pm 19$ & $11.6 \pm 1.9$ \\
\hline 0.50 & 4900 & 543 & $229.9 \pm 2.3$ & $0.44 \pm 0.05$ & 0.20 & 0.48 & 0.764 & $54 \pm 22$ & $17.7 \pm 4.0$ \\
\hline 0.50 & 4900 & 614 & $240.1 \pm 2.4$ & $0.47 \pm 0.04$ & 0.18 & 0.51 & 0.842 & $71 \pm 24$ & $15.1 \pm 2.8$ \\
\hline 0.50 & 4900 & 661 & $251.3 \pm 2.5$ & $0.49 \pm 0.03$ & 0.19 & 0.51 & 0.824 & $59 \pm 20$ & $14.6 \pm 2.6$ \\
\hline 0.50 & 4900 & 708 & $258.5 \pm 2.6$ & $0.54 \pm 0.03$ & 0.18 & 0.58 & 0.889 & $65 \pm 19$ & $10.3 \pm 1.6$ \\
\hline 0.50 & 4900 & 779 & $276.6 \pm 2.8$ & $0.58 \pm 0.03$ & 0.17 & 0.60 & 0.882 & $69 \pm 20$ & $12.5 \pm 1.8$ \\
\hline 0.50 & 4900 & 850 & $275.8 \pm 3.5$ & $0.63 \pm 0.02$ & 0.17 & 0.62 & 0.875 & $71 \pm 14$ & $16.3 \pm 1.9$ \\
\hline 0.50 & 4900 & 920 & $285.6 \pm 3.6$ & $0.78 \pm 0.01$ & 0.18 & 0.67 & 0.878 & $62 \pm 11$ & $16.6 \pm 1.9$ \\
\hline
\end{tabular}




\begin{tabular}{|c|c|c|c|c|c|c|c|c|c|}
\hline \multicolumn{10}{|c|}{$8.0 \mathrm{~mm}$ diameter tube, $823 \mathrm{~mm}$ long } \\
\hline $\begin{array}{c}S_{\mathrm{r}} \\
( \pm \mathbf{0 . 0 3})\end{array}$ & $\begin{array}{c}\nabla P \\
\left(\mathrm{~Pa} \mathrm{~m}^{-1}\right) \\
( \pm 260)\end{array}$ & $\begin{array}{c}V_{G} \\
\left(\mathrm{ml} \mathrm{min}^{-1}\right) \\
( \pm 38)\end{array}$ & $\begin{array}{c}V_{L} \\
\left(\mathrm{ml} \mathrm{min}^{-1}\right)\end{array}$ & $\underset{\left(\mathrm{m} \mathrm{s}^{-1}\right)}{\mathbf{U}_{\mathrm{b}}}$ & $\begin{array}{c}\delta_{\mathbf{f}} \\
(\mathbf{m m}) \\
( \pm 0.09)\end{array}$ & $\begin{array}{c}\alpha \\
( \pm \mathbf{0 . 0 3})\end{array}$ & $\boldsymbol{\beta}$ & $\begin{array}{c}L_{\mathrm{b}} \\
(\mathbf{m m})\end{array}$ & $\begin{array}{c}L_{\mathrm{s}} \\
(\mathbf{m m})\end{array}$ \\
\hline 0.30 & 2940 & 189 & $3.3 \pm 0.1$ & $0.18 \pm 0.06$ & 0.36 & 0.68 & 0.870 & $11 \pm 1$ & $2.0 \pm 0.5$ \\
\hline 0.30 & 2940 & 260 & $10.7 \pm 0.2$ & $0.17 \pm 0.02$ & 0.37 & 0.64 & 0.847 & $16 \pm 3$ & $3.7 \pm 0.8$ \\
\hline 0.30 & 2940 & 330 & $20.1 \pm 0.2$ & $0.20 \pm 0.04$ & 0.36 & 0.65 & 0.891 & $19 \pm 3$ & $3.1 \pm 0.5$ \\
\hline 0.30 & 2940 & 401 & $34.2 \pm 0.2$ & $0.23 \pm 0.04$ & 0.34 & 0.65 & 0.855 & $39 \pm 18$ & $11.4 \pm 5.9$ \\
\hline 0.30 & 2940 & 472 & $48.6 \pm 0.3$ & $0.24 \pm 0.03$ & 0.34 & 0.66 & 0.795 & $38 \pm 15$ & $15.5 \pm 8$ \\
\hline 0.30 & 2940 & 543 & $66.0 \pm 0.4$ & $0.23 \pm 0.01$ & 0.30 & 0.65 & 0.880 & $49 \pm 20$ & $10.2 \pm 3.5$ \\
\hline 0.30 & 2940 & 614 & $83.2 \pm 0.4$ & $0.29 \pm 0.02$ & 0.30 & 0.68 & 0.893 & $84 \pm 28$ & $19.3 \pm 6.3$ \\
\hline 0.30 & 2940 & 661 & $91.0 \pm 0.5$ & $0.32 \pm 0.05$ & 0.31 & 0.68 & 0.891 & $86 \pm 28$ & $20.6 \pm 5.5$ \\
\hline 0.30 & 2940 & 708 & $99.1 \pm 0.5$ & $0.29 \pm 0.01$ & 0.31 & 0.64 & 0.897 & $75 \pm 26$ & $16.8 \pm 4.2$ \\
\hline 0.30 & 2940 & 779 & $108.8 \pm 0.6$ & $0.32 \pm 0.02$ & 0.30 & 0.68 & 0.880 & $80 \pm 25$ & $24.7 \pm 6.0$ \\
\hline 0.30 & 2940 & 850 & $115.7 \pm 0.6$ & $0.36 \pm 0.02$ & 0.30 & 0.69 & 0.925 & $93 \pm 26$ & $22.3 \pm 4.4$ \\
\hline 0.30 & 2940 & 920 & $131.6 \pm 0.7$ & $0.39 \pm 0.02$ & 0.30 & 0.70 & 0.917 & $112 \pm 29$ & $27.6 \pm 5.4$ \\
\hline 0.40 & 3920 & 236 & $42.5 \pm 0.2$ & $0.17 \pm 0.03$ & 0.31 & 0.61 & 0.824 & $20 \pm 9$ & $5.8 \pm 3.0$ \\
\hline 0.40 & 3920 & 307 & $67.9 \pm 0.2$ & $0.19 \pm 0.02$ & 0.41 & 0.61 & 0.793 & $16 \pm 4$ & $6.0 \pm 3.0$ \\
\hline 0.40 & 3920 & 378 & $87.0 \pm 0.3$ & $0.20 \pm 0.01$ & 0.27 & 0.58 & 0.774 & $22 \pm 8$ & $8.3 \pm 2.3$ \\
\hline 0.40 & 3920 & 448 & $115.1 \pm 0.4$ & $0.22 \pm 0.02$ & 0.31 & 0.54 & 0.748 & $34 \pm 18$ & $14.1 \pm 4.8$ \\
\hline 0.40 & 3920 & 472 & $121.8 \pm 0.6$ & $0.24 \pm 0.01$ & 0.35 & 0.58 & 0.827 & $45 \pm 18$ & $13.5 \pm 4.2$ \\
\hline 0.40 & 3920 & 519 & $132.7 \pm 0.5$ & $0.26 \pm 0.02$ & 0.26 & 0.57 & 0.836 & $49 \pm 17$ & $13.9 \pm 3.3$ \\
\hline 0.40 & 3920 & 590 & $155.8 \pm 0.5$ & $0.30 \pm 0.04$ & 0.29 & 0.55 & 0.782 & $37 \pm 16$ & $13.8 \pm 3.3$ \\
\hline 0.40 & 3920 & 661 & $171.3 \pm 0.6$ & $0.32 \pm 0.04$ & 0.27 & 0.59 & 0.833 & $52 \pm 18$ & $17.2 \pm 4.3$ \\
\hline 0.40 & 3920 & 708 & $194.4 \pm 1.0$ & $0.38 \pm 0.01$ & 0.26 & 0.64 & 0.789 & $46 \pm 7$ & $22.1 \pm 4.6$ \\
\hline 0.40 & 3920 & 779 & $208.7 \pm 1.1$ & $0.41 \pm 0.01$ & 0.26 & 0.65 & 0.835 & $48 \pm 6$ & $20.3 \pm 5.3$ \\
\hline 0.40 & 3920 & 850 & $222.7 \pm 1.4$ & $0.44 \pm 0.03$ & 0.27 & 0.66 & 0.819 & $45 \pm 6$ & $20.3 \pm 3.4$ \\
\hline 0.40 & 3920 & 920 & $250.3 \pm 1.6$ & $0.46 \pm 0.01$ & 0.26 & 0.67 & 0.818 & $53 \pm 8$ & $26.0 \pm 6.7$ \\
\hline 0.50 & 4900 & 142 & $31.0 \pm 0.2$ & $0.12 \pm 0.01$ & 0.15 & 0.49 & 0.660 & $8 \pm 1$ & $4.4 \pm 0.3$ \\
\hline 0.50 & 4900 & 212 & $106.5 \pm 0.5$ & $0.16 \pm 0.01$ & 0.25 & 0.49 & 0.715 & $24 \pm 12$ & $10.5 \pm 2.8$ \\
\hline 0.50 & 4900 & 283 & $134.3 \pm 0.7$ & $0.20 \pm 0.02$ & 0.34 & 0.48 & 0.703 & $28 \pm 17$ & $13.0 \pm 4.7$ \\
\hline 0.50 & 4900 & 354 & $155.4 \pm 1.0$ & $0.21 \pm 0.02$ & 0.31 & 0.47 & 0.693 & $35 \pm 17$ & $17.0 \pm 3.5$ \\
\hline 0.50 & 4900 & 425 & $175.6 \pm 1.1$ & $0.23 \pm 0.02$ & 0.29 & 0.47 & 0.718 & $31 \pm 14$ & $15.0 \pm 3.6$ \\
\hline 0.50 & 4900 & 496 & $236.0 \pm 1.5$ & $0.32 \pm 0.01$ & 0.25 & 0.55 & 0.716 & $32 \pm 3$ & $17.6 \pm 1.4$ \\
\hline 0.50 & 4900 & 566 & $263.0 \pm 1.9$ & $0.35 \pm 0.01$ & 0.27 & 0.56 & 0.759 & $39 \pm 4$ & $19.5 \pm 1.8$ \\
\hline 0.50 & 4900 & 637 & $288.1 \pm 2.1$ & $0.38 \pm 0.01$ & 0.27 & 0.58 & 0.726 & $38 \pm 4$ & $19.1 \pm 1.9$ \\
\hline 0.50 & 4900 & 708 & $307.9 \pm 2.6$ & $0.41 \pm 0.01$ & 0.27 & 0.59 & 0.745 & $39 \pm 4$ & $20.0 \pm 1.7$ \\
\hline 0.50 & 4900 & 779 & $324.5 \pm 2.7$ & $0.44 \pm 0.01$ & 0.26 & 0.60 & 0.733 & $39 \pm 4$ & $21.5 \pm 1.8$ \\
\hline 0.50 & 4900 & 850 & $334.0 \pm 2.8$ & $0.46 \pm 0.01$ & 0.27 & 0.60 & 0.788 & $42 \pm 4$ & $20.1 \pm 1.4$ \\
\hline 0.50 & 4900 & 920 & $365.9 \pm 3.7$ & $0.51 \pm 0.01$ & 0.27 & 0.62 & 0.802 & $45 \pm 5$ & $22.2 \pm 2.1$ \\
\hline
\end{tabular}




\begin{tabular}{|c|c|c|c|c|c|c|c|c|c|}
\hline \multicolumn{10}{|c|}{$9.5 \mathrm{~mm}$ diameter tube, $846 \mathrm{~mm}$ long } \\
\hline $\begin{array}{c}S_{\mathrm{r}} \\
( \pm \mathbf{0 . 0 3})\end{array}$ & $\begin{array}{c}\nabla \mathbf{P} \\
\left(\mathbf{P a} \mathbf{~ m}^{-1}\right) \\
( \pm 260)\end{array}$ & 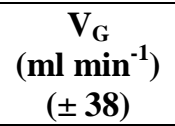 & $\begin{array}{c}V_{\mathbf{L}} \\
\left(\mathrm{ml} \mathrm{min}^{-1}\right) \\
( \pm 0.1)\end{array}$ & $\begin{array}{c}\mathbf{U}_{\mathbf{b}} \\
\left(\mathrm{m} \mathrm{s}^{-1}\right)\end{array}$ & $\begin{array}{c}\boldsymbol{\delta}_{\mathbf{f}} \\
(\mathbf{m m}) \\
( \pm \mathbf{0 . 0 8})\end{array}$ & $\begin{array}{c}\alpha \\
( \pm 0.03)\end{array}$ & $\beta$ & $\begin{array}{c}L_{\mathbf{b}} \\
(\mathbf{m m})\end{array}$ & $\begin{array}{c}L_{\mathrm{s}} \\
(\mathbf{m m})\end{array}$ \\
\hline 0.30 & 2940 & 425 & $2.5 \pm 0.2$ & $0.22 \pm 0.07$ & 0.16 & 0.77 & 0.952 & $21 \pm 5$ & $1.6 \pm 0.3$ \\
\hline 0.30 & 2940 & 472 & $2.0 \pm 0.2$ & $0.21 \pm 0.06$ & 0.23 & 0.74 & 0.901 & $32 \pm 14$ & $4.8 \pm 3.3$ \\
\hline 0.30 & 2940 & 543 & $2.6 \pm 0.2$ & $0.24 \pm 0.07$ & 0.21 & 0.74 & 0.932 & $34 \pm 11$ & $5.0 \pm 3.5$ \\
\hline 0.30 & 2940 & 614 & $3.6 \pm 0.2$ & $0.23 \pm 0.04$ & 0.29 & 0.67 & 0.864 & $37 \pm 7$ & $13.0 \pm 5.4$ \\
\hline 0.30 & 2940 & 661 & $4.3 \pm 0.2$ & $0.26 \pm 0.05$ & 0.29 & 0.68 & 0.862 & $54 \pm 17$ & $18.8 \pm 7.1$ \\
\hline 0.30 & 2940 & 708 & $4.8 \pm 0.2$ & $0.32 \pm 0.08$ & 0.38 & 0.65 & 0.847 & $61 \pm 17$ & $27.3 \pm 10.5$ \\
\hline 0.30 & 2940 & 779 & $6.8 \pm 0.2$ & $0.25 \pm 0.02$ & 0.42 & 0.64 & 0.819 & $74 \pm 23$ & $38.0 \pm 15.9$ \\
\hline 0.30 & 2940 & 850 & $12.3 \pm 0.2$ & $0.33 \pm 0.05$ & 0.36 & 0.66 & 0.848 & $74 \pm 18$ & $29.0 \pm 10.8$ \\
\hline 0.30 & 2940 & 920 & $43.0 \pm 0.3$ & $0.32 \pm 0.03$ & 0.36 & 0.68 & 0.884 & $98 \pm 23$ & $32.9 \pm 10.2$ \\
\hline 0.40 & 3920 & 236 & $2.1 \pm 0.2$ & $0.19 \pm 0.06$ & 0.26 & 0.53 & 0.722 & $24 \pm 8$ & $14.1 \pm 7.5$ \\
\hline 0.40 & 3920 & 307 & $1.3 \pm 0.2$ & $0.27 \pm 0.09$ & 0.25 & 0.52 & 0.611 & $30 \pm 9$ & $30.2 \pm 15.1$ \\
\hline 0.40 & 3920 & 378 & $3.4 \pm 0.2$ & $0.29 \pm 0.09$ & 0.36 & 0.54 & 0.677 & $35 \pm 17$ & $23.8 \pm 11.6$ \\
\hline 0.40 & 3920 & 425 & $9.4 \pm 0.2$ & $0.17 \pm 0.02$ & 0.29 & 0.54 & 0.693 & $75 \pm 33$ & $46.5 \pm 15.9$ \\
\hline 0.40 & 3920 & 472 & $19.2 \pm 0.2$ & $0.22 \pm 0.08$ & 0.29 & 0.55 & 0.661 & $67 \pm 30$ & $48.9 \pm 16.4$ \\
\hline 0.40 & 3920 & 543 & $36.7 \pm 0.3$ & $0.20 \pm 0.01$ & 0.28 & 0.60 & 0.788 & $78 \pm 25$ & $37.9 \pm 8.4$ \\
\hline 0.40 & 3920 & 614 & $66.1 \pm 0.4$ & $0.24 \pm 0.02$ & 0.32 & 0.60 & 0.759 & $79 \pm 22$ & $37.0 \pm 10.5$ \\
\hline 0.40 & 3920 & 661 & $80.4 \pm 0.6$ & $0.26 \pm 0.01$ & 0.28 & 0.61 & 0.772 & $79 \pm 19$ & $46.8 \pm 11.0$ \\
\hline 0.40 & 3920 & 708 & $94.5 \pm 0.7$ & $0.26 \pm 0.02$ & 0.30 & 0.61 & 0.773 & $80 \pm 22$ & $35.2 \pm 10.5$ \\
\hline 0.40 & 3920 & 779 & $116.4 \pm 1$ & $0.28 \pm 0.02$ & 0.31 & 0.60 & 0.781 & $76 \pm 23$ & $41.2 \pm 13.9$ \\
\hline 0.40 & 3920 & 850 & $131.9 \pm 1.1$ & $0.29 \pm 0.02$ & 0.28 & 0.63 & 0.773 & $73 \pm 16$ & $43.8 \pm 9.0$ \\
\hline 0.40 & 3920 & 920 & $171.0 \pm 1.4$ & $0.32 \pm 0.02$ & 0.27 & 0.63 & 0.756 & $88 \pm 19$ & $46.3 \pm 11$ \\
\hline 0.50 & 4900 & 189 & $23.4 \pm 0.2$ & $0.19 \pm 0.09$ & 0.28 & 0.44 & 0.657 & $100 \pm 50$ & $57.2 \pm 20.9$ \\
\hline 0.50 & 4900 & 260 & $21.3 \pm 0.2$ & $0.19 \pm 0.05$ & 0.26 & 0.50 & 0.618 & $35 \pm 11$ & $27.0 \pm 10.0$ \\
\hline 0.50 & 4900 & 330 & $42.1 \pm 0.3$ & $0.21 \pm 0.07$ & 0.31 & 0.49 & 0.579 & $32 \pm 9$ & $28.3 \pm 8.4$ \\
\hline 0.50 & 4900 & 401 & $84.2 \pm 0.6$ & $0.19 \pm 0.04$ & 0.31 & 0.50 & 0.680 & $63 \pm 31$ & $37.2 \pm 12.6$ \\
\hline 0.50 & 4900 & 472 & $111.4 \pm 0.8$ & $0.21 \pm 0.06$ & 0.38 & 0.49 & 0.650 & $43 \pm 17$ & $36.0 \pm 12.2$ \\
\hline 0.50 & 4900 & 543 & $148.0 \pm 1.1$ & $0.23 \pm 0.02$ & 0.33 & 0.53 & 0.655 & $54 \pm 8$ & $38.5 \pm 6.5$ \\
\hline 0.50 & 4900 & 614 & $187.7 \pm 1.6$ & $0.29 \pm 0.02$ & 0.34 & 0.53 & 0.673 & $51 \pm 10$ & $33.1 \pm 6.8$ \\
\hline 0.50 & 4900 & 661 & $206.8 \pm 2.1$ & $0.28 \pm 0.04$ & 0.34 & 0.53 & 0.624 & $48 \pm 8$ & $39.7 \pm 7.0$ \\
\hline 0.50 & 4900 & 708 & $227.3 \pm 2.3$ & $0.29 \pm 0.02$ & 0.35 & 0.54 & 0.683 & $47 \pm 7$ & $34.5 \pm 6.9$ \\
\hline 0.50 & 4900 & 779 & $253.3 \pm 2.5$ & $0.31 \pm 0.01$ & 0.36 & 0.54 & 0.673 & $44 \pm 9$ & $36.3 \pm 9.4$ \\
\hline 0.50 & 4900 & 850 & $267.0 \pm 3.3$ & $0.31 \pm 0.01$ & 0.36 & 0.55 & 0.690 & $53 \pm 8$ & $38.8 \pm 8.5$ \\
\hline 0.50 & 4900 & 920 & $316.6 \pm 4.0$ & $0.37 \pm 0.02$ & 0.36 & 0.57 & 0.762 & $58 \pm 11$ & $30.5 \pm 6.0$ \\
\hline
\end{tabular}

The complete set of measured parameters is reproduced in this appendix for reference, and to facilitate the use and evaluation of these results in future two-phase flow investigations. 


\section{APPENDIX B: VALIDATION OF INTERFACE IDENTIFICATION APPROACH}

In the high-speed image analysis procedure (Section 3.1), Taylor bubble liquid-gas interface positions were determined as the minimum intensity pixels on both sides of the tube axis for each row in collected photographs. Photorealistic ray-tracing simulations were conducted in the PhotoView 360 software package (Dassault Systems, 2013) to evaluate this approach, and determine the potential for optical distortion due to refraction effects. This study focused on the $6.0 \mathrm{~mm}$ ID test section ( $8.0 \mathrm{~mm}$ OD) because it had the greatest relative wall thickness and smallest predicted liquid film thickness $(\sim 0.2 \mathrm{~mm})$, and thus the greatest potential for distortion. The test section and lightbox were modeled with representative indices of refraction (Fig. 16). Uniform diffuse luminosity was specified on the rear wall of the lightbox, representative of the behavior of the backlit diffuser sheet employed in the experimental facility. Renderings were produced for a simulated small-angle-lens camera ( $3.6^{\circ}$ view angle) 0.50 meters from the test section.

High-resolution (4 MPx) renderings were generated for the $6.0 \mathrm{~mm}$ ID tube with air fill (gasonly), water fill (liquid-only), and a $0.2 \mathrm{~mm}$ thick uniform water film on the inside tube wall. This last case is representative of the predicted film thicknesses for this test section. Experimentally

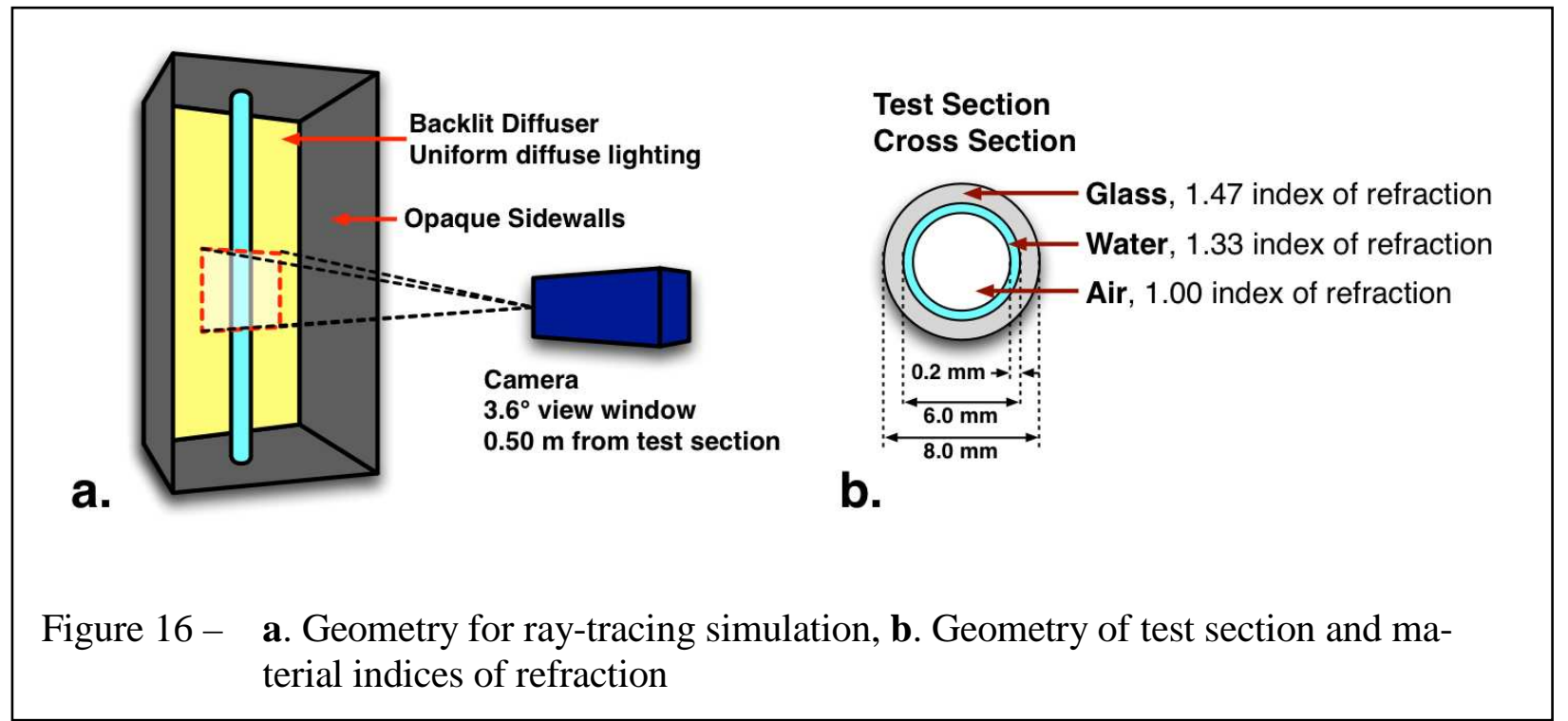




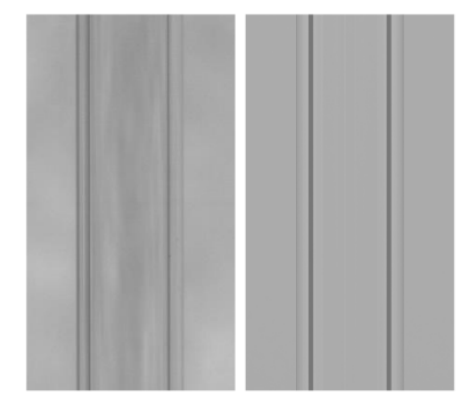

a. Photograph

Simulation

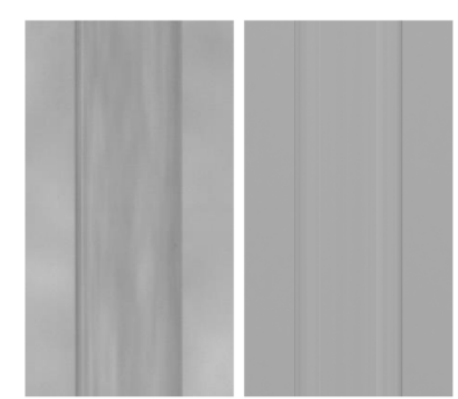

b. Photograph

Simulation

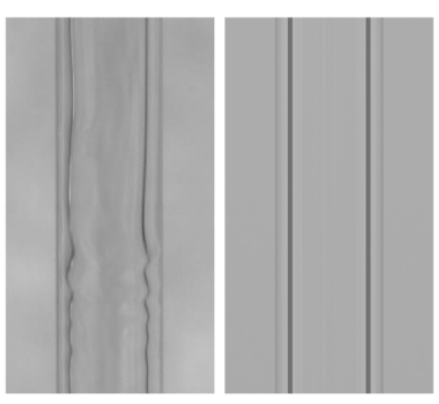

C. Photograph

Figure 17 - Comparison of experimental photographs and ray tracing simulations for: $\mathbf{a}$. air-filled tube (gas only), b. liquid-filled tube (liquid-only), c. annular water film around an elongated air bubble

collected photographs and renderings are presented side-by-side for these cases in Fig. 17. These images appear qualitatively similar, except that the texture of the experimental diffuser sheet was not incorporated in the simulation cases.

The apparent liquid-film thickness for the simulation case was evaluated using the same minimum pixel-intensity approach employed to analyze the experimental videos (Section 3.1). For this image, the average evaluated film thickness was $0.204 \mathrm{~mm}$ with a standard deviation of $0.009 \mathrm{~mm}$ over the 2048 pixel rows. The resulting error in measured film thickness $(0.004 \mathrm{~mm})$ is significantly less than the apparent rendering pixel dimension $\left(0.014 \mathrm{~mm} \mathrm{px}^{-1}\right)$. This result indicates that the reported experimental uncertainty of \pm 1 pixel $(0.07 \mathrm{~mm})$ is sufficient to account for optical distortion, and provides validation for the liquid-gas interface identification approach employed here. Representative normalized pixel-row intensity profiles are presented for the experimental and simulation cases in Fig. 18. These profiles display similar characteristics, although the wavy film is asymmetric for this row of the experimental image. Additionally, the experimental intensity profile is somewhat noisier, possibly due to the texture of the backlit diffuser sheet wall in the lightbox. 


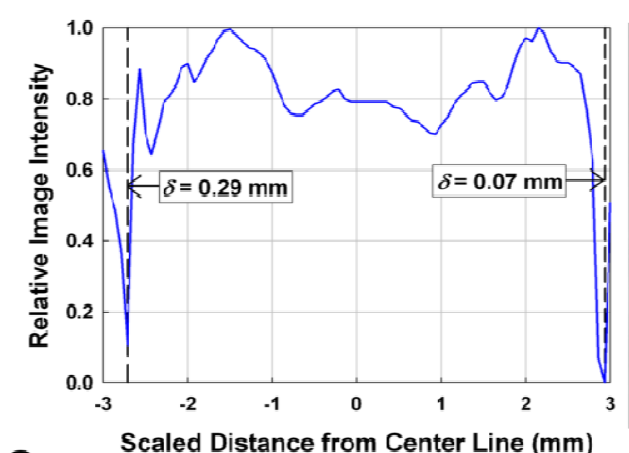

a.

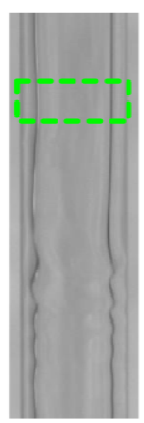

b.

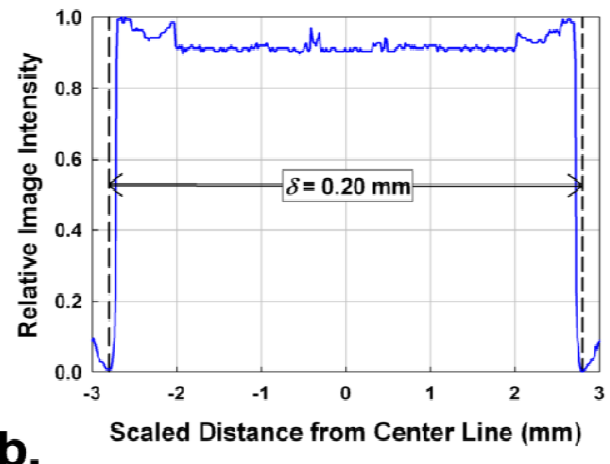

Figure 18 - Comparison of pixel intensities in indicated image regions and identified interface locations for: a. experimental photograph, b. Ray-tracing simulation 